D. N. Sarkhel, Department of Mathematics, University of Kalyani, Kalyani 741235, West Bengal, India

\title{
A UNIFIED SCALE OF ABSOLUTE AND NON-ABSOLUTE INTEGRALS
}

\begin{abstract}
In this paper a Riemann scale of integrals is defined depending on the concept of sifting. Depending on the choice of sifting, the integral defined is equivalent to various classical integrals. The associated concepts of generalized absolute continuity are also defined and used to obtain descriptive definitions of the integrals introduced.
\end{abstract}

\section{Introduction}

There have been several interesting attempts to unify and generalize the various known non-absolute integrals on the real line, [3, 4, 20, 21, 22, 24], using the methods of Denjoy and Perron. Riemann type definitions of the absolute (Lebesgue) integral $[5,8,16]$, of the Denjoy-Perron integral $[9,13,14,15]$ and of the general Denjoy integral [12] are available. In [25, 26] the KurzweilHenstock integral is studied in terms of an integration basis and a local system with the partitioning property. However the partitioning property is not available in many interesting cases. Also, the literature of multi-dimensional non-absolute integrals is growing. See references in $[6,7,17,18]$.

In this paper we define a Riemann scale of integrals in $\mathbb{R}^{r}, r \geq 1$, the $\mathcal{S}$-integral, involving the parameter of regularity of intervals and using the unifying concept of a sifting $S$, a simple class of point-interval functions, $\S 3$. The $\mathcal{S}$-integral brings all the above integrals, and many other, under one definition and one theory. The integral is defined by a new approach, simply by breaking the interval of integration into countably many subsets.

The $\mathcal{S}$-integral is additive in every expected sense, $\S 4$. Known convergence theorems are derived from two new ones in the form of necessary and sufficient

Key Words: Riemann integral, sifting, parameter of regularity

Mathematical Reviews subject classification: Primary 26A39; Secondary 26A42

Received by the editors April 14, 1993 
conditions, $\S 5$. After a detailed study of certain generalizations of absolute continuity and derivative, $\S 6$, we obtain a descriptive characterization of the $\mathcal{S}$-integral and its relation with absolute and non-absolute integrals, $\S 7$. One simple choice of $S$ gives an integral that is shown to be equivalent to the Lebesgue integral. In the one-dimensional case, $\S 8$, another choice of $S$ gives an integral that is shown to be equivalent to the restricted Denjoy integral, and for another choice, to the general Denjoy integral. In general the integrals of the $\mathcal{S}$-scale are shown to be co-extensive with the $(\mathcal{T P})$ - and $(\mathcal{T D})$ - integrals of the $\mathcal{T}$-scale, $[20,21]$.

Questions concerning a Fubini theorem and a Divergence theorem etc. remain open.

The author wishes to thank a referee for remarks about the original onedimensional version of this paper.

\section{Notation and Preliminaries}

In this section we construct a language for the present theory and obtain some elementary geometric results.

Throughout $\mathbb{R}$ will denote the real line, $r \geq 1$ a fixed integer and $Y=\mathbb{R}^{r}$, Euclidean r-space. The $i$ th coordinate of a point $y$ in $Y$ will be denoted by $y_{i}$, so that $\underline{y}=\left(y_{1}, \ldots, y_{r}\right)$. For convenience we will use the metric $d$ in $Y$ defined by

$$
d(\underline{x}, \underline{y})=\max \left\{\left|x_{i}-y_{i}\right| ; 1 \leq i \leq r\right\} .
$$

Unless otherwise implied a set $E$ will always be a subset of $Y,|E|$ will denote its outer Lebesgue measure, $\stackrel{\circ}{E}$ its interior, $\bar{E}$ its closure and $d(E)$ its diameter, with $d(\emptyset)=0 . \quad d(X, E)$ is the distance of the set $X$ from the set $E$, with $d(\emptyset, E)=\infty$.

The term interval by itself shall always mean a bounded, closed, nondegenerate interval in $Y$, say $I$, written $I=[\underline{a}, \underline{b}]$, where $\underline{a}$ and $\underline{b}$ are the principal vertices of $I$, so that

$$
I=\left\{\underline{y} ; \underline{y} \in Y, a_{i} \leq y_{i} \leq b_{i}, 1 \leq i \leq r\right\} .
$$

Clearly $d(I)=b_{i}-a_{i}$ for at least one $i$. Given $i$ the two sets $\left\{\underline{y} ; \underline{y} \in I, y_{i}=a_{i}\right\}$, $\left\{\underline{y} ; \underline{y} \in I, y_{i}=b_{i}\right\}$ are the $i$ th opposite faces of $I$, that are called diametral if $b_{i}-a_{i}=d(I)$. If $b_{1}-a_{1}=\cdots=b_{r}-a_{r}$, then $I$ is called a cube.

Two intervals $I$ and $J$ are said to overlap if $I \cap J$ is an interval; equivalently if $\stackrel{\circ}{I} \cap \stackrel{\circ}{J} \neq \emptyset$, or $I \cap \stackrel{\circ}{J} \neq \emptyset$.

The symbols $n, J$ and $(y, J)$, even with subscripts, will always denote, respectively, a positive integer, an interval and a point-interval pair, with invariably $\underline{y}$ in $J$. Unless otherwise implied all functions will be real valued. 
A $J$, or a $(\underline{y}, J)$, is said to be:

(i) at a point $\underline{c}$ if one of the $2^{r}$ vertices of $J$ is at $\underline{c}$;

(ii) over a set $E$ if $E \cap J \neq \emptyset$, or $\underline{y} \in E$;

(iii) in a set $E$ if $J \subseteq E$;

(iv) on a set $E$ if $J$ has at least one pair of opposite diametral faces both intersecting $E$; for $r=1$ this is taken to mean that $J$ has its end-points in $E$.

A family $\left\{J_{\iota}\right\}$, or $\left\{\left(y_{l}, J_{\iota}\right)\right\}$ is said to have any one of the properties (i) to (iv) if every member of the family has that property.

A finite, possibly empty, family of non-overlapping intervals $\Delta=\left\{J_{\iota}\right\}$ is called a sub-division, in $Y$, [19, p. 165], and then for any choice of $y_{\iota}$ in $J_{\iota}$ the family $\Delta^{*}=\left\{\left(y_{\iota}, J_{\iota}\right)\right\}$ is called a division, [13, p. 41]. We will strictly follow this unique correspondence between $\Delta$ and $\Delta^{*}$, omitting only the $*$.

A $\Delta$ or $\Delta^{*}$ is said to be:

(i) of a set $E$ if $\cup \Delta=\cup_{\iota} J_{\iota}=E$;

(ii) about a set $E$ if it is over $E$ and $E \subseteq \cup \Delta$.

The volume of $\Delta$, the sum over $\Delta$ of an interval function $F$ known on $\Delta$, the sum over $\Delta^{*}$ of a point-interval function $s$ known on $\Delta^{*}$ and the Riemann sum over $\Delta^{*}$ of a point function $f$ known on $\left\{\underline{y}_{\iota}\right\}$, are defined respectively by:

$$
\begin{aligned}
|\Delta| & =\sum_{\iota}\left|J_{\iota}\right|, & F(\Delta) & =\sum_{\iota} F\left(J_{\iota}\right), \\
s\left(\Delta^{*}\right) & =\sum_{\iota} s\left(\underline{y}_{\iota}, J_{\iota}\right), & f\left(\Delta^{*}\right) & =\sum_{\iota} f\left(\underline{y}_{\iota}\right)\left|J_{\iota}\right|,
\end{aligned}
$$

where all are zero if $\Delta=\emptyset$.

Two divisions $\Delta_{0}^{*}$ and $\Delta_{1}^{*}$ are non-overlapping if no interval of $\Delta_{0}$ overlaps with any interval of $\Delta_{1}$, and then $\Delta_{0}^{*} \cup \Delta_{1}^{*}$ will be written as $\Delta_{0}^{*}+\Delta_{1}^{*}$, or as $\sum_{k} \Delta_{k}^{*}$ in extended cases. Similar notation will be used for subdivisions.

Now let $\underline{c} \in I=[\underline{a}, \underline{b}]$. For each $i, 1 \leq i \leq r$ let

$$
I_{i}= \begin{cases}{\left[a_{i}, c_{i}\right] \text { or }\left[c_{i}, b_{i}\right]} & \text { if } a_{i}<c_{i}<b_{i} \\ {\left[a_{i}, b_{i}\right]} & \text { if } c_{i}=a_{i} \text { or } c_{i}=b_{i} .\end{cases}
$$

Then $J=I_{1} \times \cdots \times I_{r}$ is a subinterval of $I$ with a vertex at $\underline{c}$. There are $2^{m}$ such intervals, $0 \leq m \leq r$, that together form a subdivision of $I$. Note that 
$m=0$ iff $I$ is at $\underline{c}$, and $m=r$ iff $\underline{c} \in \stackrel{\circ}{I}$. We will call this a subdivision of $I$ at $\underline{c}$. Using this technique we easily obtain the following two lemmas.

Lemma 2.1. The greatest number of mutually non-overlapping intervals having at least one common point $\underline{c}$ is $2^{r}$.

Lemma 2.2. Every subdivision $\Delta$ in an interval $I$ can be extended to a subdivision $\Delta+\Delta^{\prime}$ of $I$.

Proof. Hint: Consider the super-imposition of the subdivisions of $I$ at the vertices of members of $\Delta \cup\{I\}$.

Lemma 2.3. An interval $J$ is on $\overline{E \cap J}$ for a set $E$ iff we have $d(E \cap J)=d(J)$.

Proof. Let $A=E \cap J$, when $\bar{A} \subseteq J$. The 'only if' part is obvious from $d(A)=d(\bar{A})$.

Next, since $\bar{A}$ is closed and bounded and since $d(A)=d(J)>0$, there must exist points $\underline{u}, \underline{v}$ in $\bar{A}$, and an index $i$, such that

$$
v_{i}-u_{i}=d(\underline{u}, \underline{v})=d(\bar{A})=d(A)=d(J) .
$$

Writing $J=[\underline{a}, \underline{b}]$, we have

$$
a_{i} \leq u_{i}<v_{i} \leq b_{i}, \quad b_{i}-a_{i} \leq d(J)=v_{i}-u_{i},
$$

and so $u_{i}=a_{i}, v_{i}=b_{i}$ and $b_{i}-a_{i}=d(J)$. So the points $\underline{u}, \underline{v}$ of $\bar{A}$ lie on the $i$ th opposite faces of $J$, which are diametral. So $J$ is on $\bar{A}$.

The quantity $Q(J)=|J| d(J)^{-r}$ is called the parameter of regularity of the interval $J$; it is 1 if $J$ is a cube; [19, p. 106].

The paper is based on the following two lemmas.

Lemma 2.4. Given a set $E$ and $a \delta, 0<\delta<1$, every subdivision $\Delta$ in an interval $I$ can be extended to a subdivision $\Delta+\Delta^{\prime}$ of I such that, if $J \in \Delta^{\prime}$ and $E \cap J \neq \emptyset$, then $Q(J)>1-\delta$ and $J \subseteq J_{i}$ for some $J_{i}$ at a point $\underline{c}_{i}$ of $E$ with $d\left(J_{i}\right)<\delta$.

Proof. Using Lemma 2.2 we easily obtain a subdivision $\Delta+\left\{I_{i}\right\}$ of $I$ such that $d\left(I_{i}\right)<\delta$ for all $i$. For each $i$ let $\left\{I_{i j}\right\}$ be the subdivision of $I_{i}$ at a point $\underline{c}_{i}$ of $I_{i}$, where $\underline{c}_{i}$ is also in $E$ whenever $E \cap I_{i} \neq \emptyset$. By a result of Pfeffer, [17, 2.3 , p. 667], there is a subdivision $\Delta_{i j}$ of $I_{i j}$ such that $Q(J)>1-\delta$ for all $J$ in $\Delta_{i j}$. The result then follows by taking $\Delta^{\prime}=\sum_{i, j} \Delta_{i j}$.

Lemma 2.5. For any interval $I$ and any set $E$ with $d(E \cap I)>0$, there is an interval $J$ on $\overline{E \cap J}$ such that $E \cap I \subseteq J \subseteq I$ and $Q(J) \geq Q(I)$. In particular any neighborhood of any limit point $\underline{y}$ of $E$ contains a cube $J$ on $\overline{E \cap J}$ with $\underline{y}$ in $J$. 
Proof. Let $I=[\underline{a}, \underline{b}]$ and $A=E \cap I$. Since $\bar{A} \subseteq I, \bar{A} \neq \emptyset$, for each $i, 1 \leq i \leq r$, there are points $\underline{a}(i), \underline{b}(i)$ in $\bar{A}$ such that

$$
a_{i} \leq a_{i}(i) \leq x_{i} \leq b_{i}(i) \leq b_{i} \quad \forall \underline{x} \in A .
$$

Let $J=[\underline{c}, \underline{c}+\underline{h}]$ where for $1 \leq i \leq r$,

$$
h_{i}=\min \left\{b_{i}-a_{i}, d(A)\right\} \text { and } c_{i}=\min \left\{b_{i}-h_{i}, a_{i}(i)\right\} .
$$

Clearly for each $i, h_{i}>0$ and we now show that

$$
a_{i} \leq c_{i} \leq a_{i}(i) \leq b_{i}(i) \leq c_{i}+h_{i} \leq b_{i} .
$$

First consider the case $h_{i}=b_{i}-a_{i}$. Then $b_{i}-h_{i}=a_{i} \leq a_{i}(i)$, by (1). So $c_{i}=b_{i}-h_{i}=a_{i}$, which by (1) implies (2).

Next let $h_{i}=d(A) \leq b_{i}-a_{i}$ and $c_{i}=b_{i}-h_{i} \leq a_{i}(i)$. Then clearly (1) implies (2).

Finally let $h_{i}=d(A)$ and $c_{i}=a_{i}(i)<b_{i}-h_{i}$. Since $b_{i}(i)-a_{i}(i) \leq d(\bar{A})=$ $d(A)$, we again get $(2)$ using (1).

Now, from (1) and (2), we see that $E \cap I=A \subseteq J \subseteq I$. Again, since by definition $h_{i} \leq d(A)$ for all $i$, we have $d(J) \leq d(A)$; so because $A=A \cap J=$ $E \cap I \cap J=E \cap J \subseteq J$, it follows that $d(J)=d(A)=d(E \cap J)$. Hence, by Lemma $2.3 J$ is on $\overline{E \cap J}$. Also, since $d(A) \leq d(I)$ and $b_{i}-a_{i} \leq d(I)$, we clearly have $h_{i} \geq\left(b_{i}-a_{i}\right) d(A) / d(I)$ for all $i$. Therefore $|J| \geq|I|(d(A) / d(I))^{r}$, which by $d(A)=d(J)$ gives $Q(J) \geq Q(I)$.

As in $[20,21,22]$, the following concepts will be very convenient. A sequence of sets $\mathfrak{E}=\left\{E_{n}\right\}_{n=1}^{\infty}$ whose union is $E$ is called an $E$-form with parts $E_{n}$; if further each part $E_{n}$ is closed in $E$, measurable in $E$, disjoint from all other parts, that is $E_{m} \cap E_{n}=\emptyset, m \neq n$, then the $E$-form is said to be closed, measurable, disjoint, respectively. An increasing $E$-form is called an $E$-chain.

We also need the filtering down principle, the (FDP). Let $\mathfrak{E}^{i}$ be some $E^{i}$ form, $i=0,1, \ldots$. If for each $n_{i}$ there is an $n_{0}$ such that $E_{n_{i}}^{i} \subseteq E_{n_{0}}^{0}$, then $\mathfrak{E}^{i}$ is said to be finer than $\mathfrak{E}^{0}$. In any case, by $\mathfrak{E}^{0} E^{1}$ we mean the $E^{0} \cap E^{1}$-form $\left\{E_{n}^{0} \cap E^{1}\right\}$, which is finer than $\mathfrak{E}^{0}$. Also, by $\mathfrak{E}=\mathfrak{E}^{0} \mathfrak{E}^{1}$ we mean any definite enumeration $\mathfrak{E}=\left\{E_{n}\right\}_{n=1}^{\infty}$ of the indexed family of sets $\left\{E_{j}^{0} \cap E_{k}^{1} ; j, k=1, \ldots\right\}$. Then $\mathfrak{E}$ is an $E^{0} \cap E^{1}$-form finer than both $\mathfrak{E}^{0}, \mathfrak{E}^{1}$.

\section{Regularity, Sifters and Sifting}

In this section we introduce our unifying concept of a sifting, a phase in the scale of integration, and construct divisions of the desired type regulated mainly by sets and also by members of siftings, called sifters. 
A sifter, in $Y$, is a non-negative function $s$, defined for all $(y, J)$ which is fine in the sense that, for every $\eta>0$ and every $(\underline{y}, J)$, there is a $J_{1}$ at $\underline{y}$ such that $J_{1} \subseteq J$ and $s\left(\underline{y}, J_{1}\right)<\eta$. Recall that every $J$ with $\underline{y} \in \stackrel{\circ}{J}$ subdivides into $2^{r}$ intervals at $y$.

A sifting, in $Y$, is a non-empty class of sifters $\mathcal{S}$ such that, if $s_{0}$ and $s_{1}$ belong to $\mathcal{S}$, then

(i) $\max \left\{s_{0}, s_{1}\right\}$ is a sifter, fineness has to be satisfied, and it belongs to $\mathcal{S}$;

(ii) if $s$ is any sifter for which there is a constant $c>0$ such that for each $y$ in $Y$ we have $s(\underline{y}, J)=c s_{0}(\underline{y}, J)$ for all $J$ at $\underline{y}$ in some neighborhood of $\underline{y}$, then $s$ belongs to $\mathcal{S}$.

Now take any set $E$, any $E$-form $\mathfrak{E}=\left\{E_{n}\right\}$, any subdivision $\Delta$, any division $\Delta^{*}$ and any sifter $s$. A $J$, or a $(y, J)$, is called E-regular if it is on $\overline{E \cap J}$, (recall (2.3)), over $E$, and in some $J_{1}$ at a point $\underline{y}_{1}$ of $E$ with $d\left(J_{1}\right)<d(E)$, (so $d(J)<d(E)$ ), and further $Q(J)>1-d(E)$, (redundant if $r=1$ ).

Note that if $E \subseteq A$, then, in either case, $E$-regular implies $A$-regular. In certain cases the converse is also true for $J$. One such case is of special interest.

We define the star-closure of $E$ to be the set $E^{+}$of all points $y$ in $Y$ such that every interval containing $y$ intersects $E$. Then $E \subseteq E^{+} \subseteq \bar{E}$, $d(E)=d\left(E^{+}\right)=d(\bar{E}), \overline{E^{+}}=\bar{E}, \bar{E} \backslash E^{+} \mid=0$, (for $r=1, \bar{E} \backslash E^{+}$is countable), $E^{+}$is measurable being almost closed, $E^{++}=E^{+}$and $H^{+} \subseteq E^{+}$ if $H \subseteq E$.

Consider any $E^{+}$-regular $J$. Clearly $J$ is on $\overline{E \cap J}$ and over $E$. Again, $J$ is in some $J_{1}$ at a point $\underline{y}_{1}$ of $E^{+}$with $d\left(J_{1}\right)<d\left(E^{+}\right)=d(E)$. Since every interval at $\underline{y}_{1}$ "opposite" to $J_{1}$ contains a point of $E$, clearly there is such a point $\underline{y}_{2}$ of $\bar{E}$ and a $J_{2}$ at $\underline{y}_{2}$ such that $J \subseteq J_{1} \subseteq J_{2}$ and $d\left(J_{2}\right)<d(E)$. Finally, $Q(J)>1-d\left(E^{+}\right)=1-\bar{d}(E)$. Hence every $E^{+}$-regular $J$ is $E$-regular.

In general, $E^{+}$does not have this property for $(\underline{y}, J),\left((\underline{y}, J)\right.$ over $E^{+}$does not imply $(y, J)$ over $E)$.

Next, a $\bar{J}$, or a $(\underline{y}, J)$ is called $\mathfrak{E}$-regular if it is $E_{n}$-regular for some $n$. The $\Delta$, or $\Delta^{*}$, is called $\mathfrak{E}$-regular, E-regular if every member of it is $\mathfrak{E}$-regular, E-regular.

The division $\Delta^{*}$ is called $s$-thin if $s\left(\Delta^{*}\right)<1$.

If $\Delta^{*}=\Delta_{0}^{*}+\Delta_{1}^{*}$ where $\Delta_{0}^{*}$ is $\mathfrak{E}$-regular and $\Delta_{1}^{*}$ is $s$-thin, then $\Delta^{*}$ is called a $\mathfrak{T}$-division where $\mathfrak{T}=(\mathfrak{E}, s)$.

Finally, for $\mathfrak{T}=(\mathfrak{E}, s), \Delta^{*}$ is called a $\mathfrak{T}$-regular division for a set $A$ if $\Delta^{*}$ is $\mathfrak{E}$-regular and if it can be extended to a division $\Delta^{*}+\Delta_{1}^{*}$ of $A$, so that $\Delta^{*}$ is in $A$, such that $\Delta_{1}^{*}$ is $s$-thin. 
Thus the $\mathfrak{E}$-regular part of any $\mathfrak{T}$-division of a set $A$ is necessarily a $\mathfrak{T}$ regular division for $A$, but not conversely.

Such partial divisions will in fact determine our integrals; compare [8]. While for both absolute and non-absolute integrals we shall use $(y, J)$ with $\underline{y}$ in $J$ in a unified manner, in McShane's definition of the absolute integral $\bar{y}$ need not be in $J,[8,16]$.

Now we call $\mathfrak{T}=(\mathfrak{E}, s)$ a trimmer and call it finer than another trimmer $\mathfrak{T}_{0}=\left(\mathfrak{E}_{0}, s_{0}\right)$ if $\mathfrak{E}$ is finer than $\mathfrak{E}_{0}$, and $s \geq s_{0}$. Then the following basic facts are obvious from the definitions.

Lemma 3.1. Let $\mathfrak{T}=(\mathfrak{E}, s)$ be a trimmer finer than the trimmer $\mathfrak{T}_{0}=$ $\left(\mathfrak{E}_{0}, s_{0}\right)$. Then every $\mathfrak{E}$-regular $J$, or $(y, J)$, or subdivision, or division is $\mathfrak{E}_{0}$-regular; every $s$-thin division is $s_{0}$-thin, every $\mathfrak{T}$-division is a $\mathfrak{T}_{0}$-division, and every $\mathfrak{T}$-regular division for a set $A$ is a $\mathfrak{T}_{0}$-regular division for $A$.

We now show how to construct our regulated divisions.

Lemma 3.2. Let $\mathfrak{T}=\left(\mathfrak{E}^{0}, s\right)$ where $\mathfrak{E}^{0}=\left\{E_{j}^{0}\right\}_{j=1}^{\infty}$ is some A-form and $s$ is a sifter. Let $I$ be an interval, $E$ a subset of $A \cap I, \mathfrak{E}^{1}=\left\{E_{j}^{1}\right\}_{j=1}^{\infty}=\mathfrak{E}^{0} E, E_{0}=\emptyset$, $E_{n}=\cup_{j=1}^{n} E_{j}^{1}$. Then for each $n$ there is a $\mathfrak{T}$-division $\Delta_{n-1}^{*}$ about $E_{n-1}$ in $I$, with $\Delta_{n-1}^{*} \subseteq \Delta_{n}^{*}$ for all $n$; and if $E=I$, then some $\Delta_{m}^{*}$ is a division of $I$, and then the $\mathfrak{E}^{0}$-regular part of $\Delta_{m}^{*}$ is a $\mathfrak{T}$-regular division for $I$.

Proof. For this proof we will denote by $\Delta^{0}$ the family of non $\mathfrak{E}^{0}$-regular members of a division $\Delta^{*}$. Let $\delta_{j}=d\left(E_{j}^{0}\right)$ if $d\left(E_{j}^{0}\right)>0$ and $\delta_{j}=1$ otherwise, $j=1, \ldots$. Clearly $\Delta_{0}^{*}=\emptyset$ and we define the $\Delta_{n}^{*}, n=1, \ldots$ by recursion. Assume that for some $n \geq 1$ we have defined $\Delta_{n-1}^{*}$. Write $\Delta_{n 0}^{*}=\Delta_{n-1}^{*}$. Since it is a $\mathfrak{T}$-division, $\eta=1-s\left(\Delta_{n 0}^{0}\right)>0$.

We will successively extend $\Delta_{n 0}^{*}$ to a division

$$
\Delta_{n}^{*}=\sum_{k=0}^{n} \Delta_{n k}^{*}=\Delta_{n-1}^{*}+\Delta_{n 1}^{*}+\cdots+\Delta_{n n}^{*}
$$

in $I$, with $s\left(\Delta_{n j}^{0}\right)<\eta / n$, for $1 \leq j \leq n, n$ fixed.

Suppose that $\sum_{k=0}^{j-1} \Delta_{n k}^{*}$ has been defined for some $j, 1 \leq j \leq n$. By Lemma $2.4 \sum_{k=0}^{j-1} \Delta_{n k}$ can be extended to a subdivision of $I$,

$$
\sum_{k=0}^{j-1} \Delta_{n k}+\Delta_{n j}^{\prime}, \Delta_{n j}^{\prime}=\left\{I_{i}\right\}, \text { say }
$$

such that, for each $I_{i}$ intersecting $E_{j}^{1}$ we have

$$
Q\left(I_{i}\right)>1-\delta_{j}, \text { and } I_{i} \subseteq J_{i}
$$


for some $J_{i}$ at a point $\underline{c}_{i}$ of $E_{j}^{1}$ with $d\left(J_{i}\right)<\delta_{j}$. We then form a division $\Delta_{n j}^{*}$ by choosing one and only one $(\underline{y}, J)$ corresponding to each $I_{i}$ intersecting $E_{j}^{1}$ as follows.

If $E_{j}^{1} \cap I_{i}$ is a singleton $\{\underline{y}\}$, by the fineness of $s$ there is a $J$ at $\underline{y}$ such that $J \subseteq I_{i}$ and $s(y, J)<\eta /(m n)<1$, where $m$ is a positive integer exceeding the number of indices $i$. We take this $(\underline{y}, J)$ in $\Delta_{n j}^{*}$.

If $d\left(E_{j}^{1} \cap I_{i}\right)>0$, by Lemma 2.5 there is a $J$ on $\overline{E_{j}^{1} \cap J}$ with $E_{j}^{1} \cap I_{i} \subseteq J \subseteq I_{i}$ and $Q(J) \geq Q\left(I_{i}\right)$. Take any point $\underline{y}$ of $E_{j}^{1} \cap I_{i}$. Then, since $E_{j}^{1} \subseteq E_{j}^{0}$ and by (4), clearly $(\underline{y}, J)$ is on $\overline{E_{j}^{0} \cap J}$, over $E_{j}^{0}$, and in $J_{i}$ which is at the point $\underline{c}_{i}$ of $E_{j}^{0}$ with $d\left(J_{i}\right)<\delta_{j}=d\left(E_{j}^{0}\right)$ and further $Q(J)>1-\delta_{j}=1-d\left(E_{j}^{0}\right)$. So $(\underline{y}, J)$ is $\mathfrak{E}^{0}$-regular, and we take it in $\Delta_{n j}^{*}$. In either case, for each $I_{i}$ intersecting $E_{j}^{1}$ we have

$$
E_{j}^{1} \cap I_{i} \subseteq J \subseteq I_{i} \text { for a unique } J \text { in } \Delta_{n j} .
$$

Also, evidently $s\left(\Delta_{n j}^{0}\right)<\sum_{i} \eta /(m n)<\eta / n$. Thus $\sum_{k=0}^{j} \Delta_{n k}^{*}$ is well-defined.

Hence we arrive at $\Delta_{n}^{*}$ by a recursion on $j$. In the last step $j=n, \Delta_{n n}^{*}$ is over $E_{n}^{1}$ and then (5) implies that $\Delta_{n}^{*}$ is about $E_{n}$, since $\Delta_{n}^{*}$ contains $\Delta_{n-1}^{*}$ which is already about $E_{n-1}$. Also, by our construction, starting with $n=1$, if $(\underline{y}, J) \in \Delta_{n}^{0}$, then $J$ is at $\underline{y}$, and further

$$
s\left(\Delta_{n}^{0}\right)=s\left(\Delta_{n 0}^{0}\right)+\sum_{j=1}^{n} s\left(\Delta_{n j}^{0}\right)<1-\eta+\sum_{j=1}^{n} \frac{\eta}{n}=1 .
$$

So $\Delta_{n}^{0}$ is $s$-thin, in a special way, and hence $\Delta_{n}^{*}$ is a $\mathfrak{T}$-division. Thus $\Delta_{n}^{*}$ has all the properties required by the lemma. This completes the recurrence on $n$, and defines $\Delta_{n}^{*}$ for all $n$.

Now write $D_{n}=\bigcup\left(\sum_{k=0}^{n} \Delta_{n k}\right)$ and $D_{n j}^{\prime}=\bigcup \Delta_{n j}^{\prime}$. Suppose that $E=I$, which requires $A \supseteq I$. Consider any $\underline{x}$ in $I$. Clearly $\underline{x}$ is in $E_{j}^{1}$ for some $j \geq 1$. Consider then any $n \geq j$. If $\underline{x} \in D_{n j}^{\prime}$, then by our construction $\underline{x} \in E_{j}^{1} \cap I_{i}$ for some $i$, and so by (5) $\underline{x} \in J$ for some $J$ in $\Delta_{n j}$. Hence, since the subdivisions $\left\{\Delta_{n j}\right\}_{n=j}^{\infty}$ are non-overlapping, Lemma 2.1 implies that $\underline{x} \notin D_{n j}^{\prime}$ for some $n>j$. Since each $D_{n j}^{\prime}$ is closed and by (3) $D_{n} \cup D_{n j}^{\prime}=I$, it follows that each $\underline{x}$ in $I$ is interior to some $D_{n}$ in $I$. Also, since $\Delta_{n}^{*} \subseteq \Delta_{n+1}^{*}$ and $D_{n}=\cup \Delta_{n}$, we have $D_{n} \subseteq D_{n+1}, n=1, \ldots$. Hence by the compactness of $I, D_{m}=I$ for some $m$ and then $\Delta_{m}^{*}$ is a division of $I$. Since further, the non $\mathfrak{E}^{0}$-regular part $\Delta_{m}^{0}$ of $\Delta_{m}^{*}$ is $s$-thin; so $\Delta_{m}^{*} \backslash \Delta_{m}^{0}$ is a $\mathfrak{T}$-regular division for $I$.

In the Kurzweil-Henstock theory one uses a gauge, namely, a positive function on $Y$. In the present general context, $Y$-forms will be suitable substitutes for gauges.

In the sequel we will need the following special results. 
Lemma 3.3. (i) For any gauge $p$ on $Y$, there is a $Y$-form $\mathfrak{E}$ such that, for each $n, d\left(E_{n}\right)<p(\underline{y})$ for all $y$ in $E_{n}$. Also, for every interval $I$ there is a division $\left\{\left(\underline{y}_{i}, J_{i}\right)\right\}$ of I such that, for each $i, J_{i}$ is at $\underline{y}_{i}$ and $d\left(J_{i}\right)<p\left(\underline{y}_{i}\right)$.

(ii) Given a subdivision $\left\{I_{i}\right\}$ of an interval $I$, there is a $Y$-form $\mathfrak{E}$ such that every $\mathfrak{E}$-regular $J$, or $(\underline{y}, J)$, in $I$ is in some $I_{i}$.

(iii) If $E \subseteq G$, where $G$ is open, then there is an $E$-form $\mathfrak{E}^{1}$ such that every $\mathfrak{E}^{1}$-regular $J$, or $(\underline{y}, J)$, is in $G$.

Proof. (i) Let $E^{i}=\{\underline{y} ; \underline{y} \in Y, p(\underline{y})>1 / i\}, i=1, \ldots$, and let $\left\{E_{j}^{i}\right\}$ be an $E^{i}$-form with $d\left(E_{j}^{i}\right)<1 / i$ for all $j=1, \ldots$ Then it suffices to take $\mathfrak{E}$ to be an enumeration of the family of sets $\left\{E_{j}^{i} ; i, j=1, \ldots\right\}$.

For the second part, take the trimmer $\mathfrak{T}=(\mathfrak{E}, s)$ where $s(\underline{y}, J)=d(J) / p(y)$. By Lemma 3.2, there is a $\mathfrak{T}$-division $\left\{\left(\underline{y}_{j}, I_{j}\right)\right\}$ of $I$. If $\left(\underline{y}_{j}, I_{j}\right)$ is $\mathfrak{E}$-regular, then it is $E_{n}$-regular for some $n$, and then $\underline{y}_{j} \in E_{n}$ and $d\left(I_{j}\right)<d\left(E_{n}\right)<p\left(\underline{y}_{j}\right)$; otherwise $s\left(\underline{y}_{j}, I_{j}\right)<1$ whence $d\left(I_{j}\right)<p\left(\underline{y}_{j}\right)$. Hence, replacing each $I_{j}$ by the subdivision of $I_{j}$ at $\underline{y}_{j}$ the resulting division $\left\{\left(\underline{y}_{i}, J_{i}\right)\right\}$ of $I$ is of the required type.

This is essentially another proof of Theorem 4.1 in [13, p. 42].

(ii) Evidently there is a gauge $p$ such that each $\left(\underline{y}_{1}, J_{1}\right)$ with $J_{1}$ at $\underline{y}_{1}$ and $d\left(J_{1}\right)<p\left(\underline{y}_{1}\right)$ is either in $Y \backslash \stackrel{\circ}{I}$ or in some $I_{i}$. Take the $Y$-form $\mathfrak{E}$ given by (i) for this $p$. Then, for every E-regular $J$, or $(y, J)$ in $I$, there is an $n$ such that $J \subseteq J_{1}$ for some $J_{1}$ at a point $\underline{y}_{1}$ of $E_{n}$ with $\bar{d}\left(J_{1}\right)<d\left(E_{n}\right)<p\left(\underline{y}_{1}\right)$, and hence, by noting that $J$ is in $I$, by definition of $p\left(\underline{y}_{1}\right)$ we must have $\bar{J} \subseteq J_{1} \subseteq I_{i}$ for some $i$.

(iii) Since $G$ is open, there is a gauge $p$ such that each $(\underline{y}, J)$ over $E$ with $d(J)<p(\underline{y})$ is in $G$. Take a $Y$-form $\mathfrak{E}$ given by (i) for this $p$. Then $\mathfrak{E}^{1}=\mathfrak{E} E$ is an $E$-form, and for every $\mathfrak{E}^{1}$-regular $J$, or $(y, J)$ (For $\mathfrak{E}^{1}$-regular $J$, we can certainly choose a $y$ so that $(y, J)$ is $\mathfrak{E}^{1}$-regular.) there is an $n$ such that $\underline{y} \in E_{n}^{1}=E_{n} \cap E$ and $d(J)<\bar{d}\left(E_{n}^{1}\right) \leq d\left(E_{n}\right)<p(\underline{y})$, and so $J \subseteq G$.

Lemma 3.4. For any set $E$ of measure zero, and any point function $f$ known on $E$ and any $\eta>0$, there is a $Y$-form $\mathfrak{E}$ such that for every $\mathfrak{E}$-regular division $\Delta^{*}$ over $E$ we have $\left|f\left(\Delta^{*}\right)\right|<\eta$.

Proof. For $k=1, \ldots$ let $G_{k}$ be an open set containing $E$ with $\left|G_{k}\right|<\eta / k 3^{k}$. We obtain a $Y$-form $\left\{E_{i}\right\}$ by requiring that $E_{1}=Y \backslash E$ and for $i>1, E_{i}$ is the set of points $\underline{y}$ of $E$ such that every $(\underline{y}, J)$ with $d(J)<1 / i$ is in some $G_{k}$ with $k>|f(\underline{y})|$. Let $\left\{E_{i j}\right\}$ be an $E_{i}$-form with $d\left(E_{i j}\right)<1 / i$ for all $j=1, \ldots$ and 
let $\mathfrak{E}$ be an enumeration of the family of sets $\left\{E_{i j} ; i, j=1, \ldots\right\}$. Obviously, $\mathfrak{E}$ is a $Y$-form.

Now, for $\mathfrak{E}$-regular $(y, J)$ over $E$, there is a set $E_{i j}$, say, with $i>1$ such that $y \in E_{i j} \subseteq E_{i}$ and $d(J)<d\left(E_{i j}\right)<1 / i$ and $J \subseteq G_{k}$ for some $k>|f(y)|$. Therefore, any $\mathfrak{E}$-regular division $\Delta^{*}$ over $E$ can be written as $\Delta^{*}=\sum \bar{\Delta}_{k}^{*}$ where for each $k$ and each $(\underline{y}, J) \in \Delta_{k}^{*}$ we have $|f(\underline{y})|<k$ and $J \subseteq G_{k}$. Since the intervals $J$ of $\Delta_{k}^{*}$ are non-overlapping, it follows that

$$
\left|f\left(\Delta^{*}\right)\right| \leq \sum|f|\left(\Delta_{k}^{*}\right) \leq \sum k\left|G_{k}\right|<\sum \frac{\eta}{3^{k}}<\eta .
$$

Notation In the sequel, $\mathcal{S}$ will be an arbitrary but fixed sifting in $Y, I$ an interval in $Y$, and $f, g, h, f_{0}, f_{1}, f_{2}, \ldots$ real valued point functions defined at least on $I$. For any $Y$-form $\mathfrak{E}$ and any sifter $s$ in $\mathcal{S}$, we will call $\mathfrak{T}=(\mathfrak{E}, s)$ an $\mathcal{S}$ - trimmer and denote by $I(\mathfrak{T})$ the collection of all $\mathfrak{T}$-regular divisions for the interval $I$. By Lemma 3.2, $I(\mathfrak{T})$ is never empty.

\section{The $\mathcal{S}$-integral}

Considering all $\mathcal{S}$-trimmers, $\mathfrak{T}=(\mathfrak{E}, \mathrm{s})$, we define

$$
\mathfrak{T}(f, I)=\sup _{\Delta^{*} \in I(\mathfrak{T})} f\left(\Delta^{*}\right), \mathcal{S}(f, I)=\inf _{\mathfrak{T}} \mathfrak{T}(f, I)
$$

and

$$
\mathfrak{T}^{o}(f, I)=\mathfrak{T}(f, I)+\mathfrak{T}(-f, I) .
$$

We call $\mathfrak{T}^{o}(f, I)$ the $\mathfrak{T}$-oscillatory sum, and $\mathcal{S}(f, I)$ and $-\mathcal{S}(-f, I)$, respectively the upper and lower $\mathcal{S}$-integrals of $f$ on $I$; and if these two are finite and equal, then $f$ is said to be $\mathcal{S}$-integrable on $I$, with definite integral $\mathcal{S}(f, I)$ written $(\mathcal{S}) \int_{I} f=\mathcal{S}(f, I)$. Obviously $(\mathcal{S}) \int_{I} f=0$ if $f=0$ on $I$.

The following line of reasoning will be our total filtering down principle, (FDP). Given any two $\mathcal{S}$-trimmers $\mathfrak{T}_{i}=\left(\mathfrak{E}_{i}, s_{i}\right), i=0,1$ by definition $s=$ $\max \left\{s_{0}, s_{1}\right\}$ is in $\mathcal{S}$. Also $\mathfrak{E}=\mathfrak{E}_{0} \mathfrak{E}_{1}$ is a $Y$-form finer than both $\mathfrak{E}_{0}$ and $\mathfrak{E}_{1}$. Thus $\mathfrak{T}=(\mathfrak{E}, \mathrm{s})$ is an $\mathcal{S}$-trimmer finer than both $\mathfrak{T}_{0}$ and $\mathfrak{T}_{1}$. Then by Lemma 3.1 we have $I(\mathfrak{T}) \subseteq I\left(\mathfrak{T}_{0}\right) \cap I\left(\mathfrak{T}_{1}\right)$, and hence

$$
-\infty<\mathfrak{T}(f, I) \leq \mathfrak{T}_{i}(f, I) \text { and } \mathfrak{T}^{o}(f, I) \leq \mathfrak{T}_{i}^{o}(f, I), i=0,1 .
$$

Then for all $\Delta^{*} \in I(\mathfrak{T})$ we have

$$
-\mathfrak{T}_{1}(-f, I) \leq-(-f)\left(\Delta^{*}\right)=f\left(\Delta^{*}\right) \leq \mathfrak{T}_{0}(f, I) .
$$

This evidently implies the following assertion. 
Theorem 4.1. (i) For any $\mathcal{S}$-trimmer $\mathfrak{T}$ and $\Delta^{*} \in I(\mathfrak{T})$,

$$
-\mathfrak{T}(-f, I) \leq-\mathcal{S}(-f, I) \leq \mathcal{S}(f, I) \leq \mathfrak{T}(f, I),
$$

and

$$
\left|\mathcal{S}(f, I)-f\left(\Delta^{*}\right)\right| \leq \mathfrak{T}^{o}(f, I) .
$$

(ii) The function $f$ is $\mathcal{S}$-integrable on I iff for each $\eta>0$ there is an $\mathcal{S}$ trimmer $\mathfrak{T}$ such that $\mathfrak{T}^{o}(f, I)<\eta$.

(iii) The function $f$ is $\mathcal{S}$-integrable on I iff there is a real number $c$ and to each $\eta>0$ there corresponds an $\mathcal{S}$-trimmer $\mathfrak{T}=(\mathfrak{E}, s)$ such that for all $\Delta^{*} \in I(\mathfrak{T})$ we have $\left|f\left(\Delta^{*}\right)-c\right|<\eta$; and then $\mathcal{S}(f, I)=c$.

Note 1. Clearly Theorem 4.1 (iii) implies a Riemann type definition of the $\mathcal{S}$-integral for vector-valued point-interval functions.

Note 2. When $f$ is $\mathcal{S}$-integrable on $I$, Theorem 4.1 (iii) implies that the value of the integral can be also approximated by Riemann sums of $f$ over full divisions of $I$. For, extending $f$ to $Y$ in any manner, let

$$
s_{0}(\underline{y}, J)=\max \left\{\frac{|f(\underline{y})|}{\eta}|J|, s(\underline{y}, J)\right\} .
$$

Clearly $s_{0}$ is a sifter, that may not belong to $\mathcal{S}$, and $\mathfrak{T}_{0}=\left(\mathfrak{E}, s_{0}\right)$ is finer than $\mathfrak{T}$. Take any $\Delta^{*} \in I\left(\mathfrak{T}_{0}\right)$. Then by Lemma $3.1 \Delta^{*} \in I(\mathfrak{T})$. Also $\Delta^{*}$ can be extended to a division $\Delta_{1}^{*}=\Delta^{*}+\Delta_{0}^{*}$ of $I$ where $\Delta_{0}^{*}$ is $s_{0}$-thin. Hence

$$
\left|f\left(\Delta_{1}^{*}\right)-c\right| \leq\left|f\left(\Delta^{*}\right)-c\right|+\left|f\left(\Delta_{0}^{*}\right)\right|<\eta+\eta s_{0}\left(\Delta_{0}^{*}\right)<\eta+\eta,
$$

which proves our assertion.

Using (FDP), from Theorem 4.1 (iii) we readily obtain the next theorem.

Theorem 4.2. The $\mathcal{S}$-integrable functions on I form a linear space, and the $\mathcal{S}$-integral is a linear functional on it.

Lemma 4.3. (i) For any subdivision $\left\{I_{i}\right\}_{i=1}^{m}$ of [in] I, any $c_{i}>0$ with $\sum_{i=1}^{m} c_{i}<1$, and any $\mathcal{S}$-trimmer $\mathfrak{T}=\left(\mathfrak{E}\right.$, s), and letting $\mathfrak{T}_{i}$ denoting the $\mathcal{S}$-trimmer $\left(\mathfrak{E}, c_{i}^{-1} s\right)$ we have

$$
\begin{gathered}
\sum_{i=1}^{m} \mathfrak{T}_{i}\left(f, I_{i}\right) \leq \mathfrak{T}(f, I) \\
{\left[\sum_{i=1}^{m} \mathfrak{T}_{i}^{o}\left(f, I_{i}\right) \leq \mathfrak{T}^{o}(f, I)\right] .}
\end{gathered}
$$


(ii) If $f$ is $\mathcal{S}$-integrable on $I$, then for each $\eta>0$ there is an $\mathcal{S}$-trimmer $\mathfrak{T}_{1}$ such that $\mathfrak{T}_{1}^{o}(f, J)<\eta$ for all $J$ in $I$.

Proof. (i) Recalling Lemma 2.2 and noting that $\mathfrak{T}_{i}^{o}\left(f, I_{i}\right) \geq 0$ and $\sum_{i=1}^{m} c_{i}<$ 1 , we can assume in both cases that $\cup_{i} I_{i}=I$.

Now any $\Delta_{i}^{*} \in I_{i}\left(\mathfrak{T}_{i}\right)$ is $\mathfrak{E}$-regular, and it can be extended to a division $\Delta_{i}^{*}+\Delta_{i 0}^{*}$ of $I_{i}$, where $\Delta_{i 0}^{*}$ is $c_{i}^{-1} s$-thin; that is $c_{i}^{-1} s\left(\Delta_{i 0}^{*}\right)<1$, or $s\left(\Delta_{i 0}^{*}\right)<c_{i}$. Write $\Delta^{*}=\sum_{i=1}^{m} \Delta_{i}^{*}$ and $\Delta_{0}^{*}=\sum_{i=1}^{m} \Delta_{i 0}^{*}$. Then $\Delta^{*}$ is E-regular and $\Delta^{*}+\Delta_{0}^{*}$ is a division of $I$, where $\Delta_{0}^{*}$ is $s$-thin because $s\left(\Delta_{0}^{*}\right)=\sum s\left(\Delta_{i 0}^{*}\right)<\sum c_{i}<1$. Therefore $\Delta^{*} \in I(\mathfrak{T})$ and hence $\sum_{i=1}^{m} f\left(\Delta_{i}^{*}\right)=f\left(\Delta^{*}\right) \leq \mathfrak{T}(f, I)$. Since $\Delta_{i}^{*} \in$ $I_{i}\left(\mathfrak{T}_{i}\right)$ is arbitrary and $\mathfrak{T}_{i}\left(f, I_{i}\right) \neq-\infty$ we have (6). Further

$$
\sum_{i=1}^{m} \mathfrak{T}_{i}^{o}\left(f, I_{i}\right)=\sum_{i=1}^{m}\left(\mathfrak{T}_{i}\left(f, I_{i}\right)+\mathfrak{T}_{i}\left(-f, I_{i}\right)\right) \leq \mathfrak{T}(f, I)+\mathfrak{T}(-f, I)=\mathfrak{T}^{o}(f, I),
$$

which is (7).

(ii) By Theorem 4.1 (ii), there is an $\mathcal{S}$-trimmer $\mathfrak{T}=(\mathfrak{E}, \mathrm{s})$ such that $\mathfrak{T}^{o}(f, I)<\eta$. Let $\mathfrak{T}_{1}=(\mathfrak{E}, 2 s)$. For any $J$ in $I$ taking $\left\{I_{1}\right\}=\{J\}$ in (i) we get $\mathfrak{T}_{1}^{o}(f, J) \leq \mathfrak{T}^{o}(f, I)<\eta$.

The upper and lower $\mathcal{S}$-integrals are nicely additive.

Theorem 4.4. Let $\left\{I_{i}\right\}_{i=1}^{m}$ be any subdivision of $I$. Then:

(i) $\mathcal{S}(f, I)=\sum_{i=1}^{m} \mathcal{S}\left(f, I_{i}\right)$, provided the sum on the right-hand side is defined.

(ii) $\mathcal{S}(f, I)$ is finite iff all of the $\mathcal{S}\left(f, I_{i}\right)$ are finite.

Proof. (i) Since $\sum_{i=1}^{m} \mathcal{S}\left(f, I_{i}\right)$ is defined, possibly infinite, by Lemma 4.3 (i) we have $\sum_{i=1}^{m} \mathcal{S}\left(f, I_{i}\right) \leq \mathfrak{T}(f, I)$ for every $\mathcal{S}$-trimmer $\mathfrak{T}$. Hence,

$$
\sum_{i=1}^{m} \mathcal{S}\left(f, I_{i}\right) \leq \mathcal{S}(f, I)
$$

For the reverse inequality, consider any $\mathcal{S}$-trimmers $\mathfrak{T}_{i}=\left(\mathfrak{E}_{i}, s_{i}\right), 1 \leq i \leq m$. By repeated use of $(\mathrm{FDP})$, there is an $\mathcal{S}$-trimmer $\mathfrak{T}_{0}=\left(\mathfrak{E}_{0}, s_{0}\right)$ finer than each $\mathfrak{T}_{i}$. Again, by Lemma 3.3 (ii) there is a $Y$-form $\mathfrak{E}^{\prime}$ such that every $\mathfrak{E}^{\prime}$-regular $(\underline{y}, J)$ in $I$ is in some $I_{i}$. Also, let $s(\underline{y}, J)=s_{0}(\underline{y}, J)$ if $(\underline{y}, J)$ is either in $Y \backslash \stackrel{\circ}{I}$ or in some $I_{i}$, and $s(\underline{y}, J)=\max \left\{1, s_{0}(\underline{y}, J)\right\}$ otherwise. The definition of a sifting evidently implies that $s \in \mathcal{S}$. Let $\mathfrak{E}=\mathfrak{E}_{0} \mathfrak{E}^{\prime}$. Then $\mathfrak{T}=(\mathfrak{E}, \mathrm{s})$ is a $\mathcal{S}$-trimmer finer than $\mathfrak{T}_{0}$, and hence finer than each $\mathfrak{T}_{i}$. 
Now, every $\Delta^{*} \in I(\mathfrak{T})$ is $\mathfrak{E}$-regular, and it can be extended to a division $\Delta^{*}+\Delta_{0}^{*}$ of $I$ where $\Delta_{0}^{*}$ is $s$-thin. Then, by the above, we can evidently write $\Delta^{*}=\sum_{i=1}^{m} \Delta_{i}^{*}$ and $\Delta_{0}^{*}=\sum_{i=1}^{m} \Delta_{i 0}^{*}$ where $\Delta_{i}^{*}+\Delta_{i 0}^{*}$ is in $I_{i}, 1 \leq i \leq m$. Since $\mathfrak{T}$ is finer than $\mathfrak{T}_{i}$, by Lemma $3.1 \Delta_{i}^{*}$ is $\mathfrak{E}_{i}$-regular and $\Delta_{i 0}^{*}$ is $s_{i}$-thin. Besides, since $\Delta+\Delta_{0}=\sum_{i=1}^{m}\left(\Delta_{i}+\Delta_{i 0}\right)$ is a subdivision of $I$, and since $\bigcup\left(\Delta_{i}+\Delta_{i 0}\right)$ is a closed set in $I_{i}$ for each $i$, clearly $\Delta_{i}^{*}+\Delta_{i 0}^{*}$ is a division of $I_{i}$. Therefore $\Delta_{i}^{*} \in I_{i}\left(\mathfrak{T}_{i}\right), 1 \leq i \leq m ;$ and so

$$
f\left(\Delta^{*}\right)=\sum_{i=1}^{m} f\left(\Delta_{i}^{*}\right) \leq \sum_{i=1}^{m} \mathfrak{T}_{i}\left(f, I_{i}\right), \Delta^{*} \in I(\mathfrak{T}) .
$$

Hence $\mathcal{S}(f, I) \leq \mathfrak{T}(f, I) \leq \sum \mathfrak{T}_{i}\left(f, I_{i}\right)$. Since the $\mathfrak{T}_{i}$ are arbitrary and since $\sum \mathcal{S}\left(f, I_{i}\right)$ is well defined, it follows that $\sum_{i=1}^{m} \mathcal{S}\left(f, I_{i}\right) \geq \mathcal{S}(f, I)$, which by (8) completes the proof of (i).

(ii) If $\mathcal{S}(f, I)$ is finite, then $\mathfrak{T}(f, I)<\infty$ for some $\mathcal{S}$-trimmer $\mathfrak{T}$. Then by Lemma 4.3 (i), $\mathcal{S}\left(f, I_{i}\right)<\infty$ for all $i$, which by $(i)$ above implies that all $\mathcal{S}\left(f, I_{i}\right)$ are finite. The converse is immediate from $(i)$.

Theorem 4.5. For any subdivision $\left\{I_{i}\right\}$ of $I$, we always have

$$
(\mathcal{S}) \int_{I} f=\sum_{i}(\mathcal{S}) \int_{I_{i}} f
$$

whenever the right-hand side or the left-hand side is defined.

Proof. If $f$ is $\mathcal{S}$-integrable on $I$, or if $f$ is $\mathcal{S}$-integrable on each $I_{i}$, then in either case by Theorem 4.4 we have

$$
\mathcal{S}(f, I)=\sum \mathcal{S}\left(f, I_{i}\right)=-\sum \mathcal{S}\left(-f, I_{i}\right)=-\mathcal{S}(-f, I),
$$

and further, recalling Theorem 4.1 (i) $-\infty<-\mathcal{S}\left(-f, I_{i}\right) \leq \mathcal{S}\left(f, I_{i}\right)<\infty$ for each $i$. Hence, clearly, in either case, all the integrals exist and the required equality holds.

We now prove an elementary but very useful result which extends the SaksHenstock lemma, [15, Theorem 3.7, p. 11].

Lemma 4.6. For any $\mathcal{S}$-trimmer, $\mathfrak{T}=(\mathfrak{E}, s)$, and any $\mathfrak{E}$-regular [s-thin] division $\left\{\left(\underline{y}_{i}, J_{i}\right)\right\}$ in $I$, we have

$$
\sum_{i}\left|\mathcal{S}\left(f, J_{i}\right)-f\left(\underline{y}_{i}\right)\right| J_{i}|| \leq \mathfrak{T}^{o}(f, I),\left[\sum_{i}\left|\mathcal{S}\left(f, J_{i}\right)\right| \leq \mathfrak{T}^{o}(f, I)\right] .
$$


Proof. In the first case, choose $c_{i}>0$ with $\sum_{i} c_{i}<1$, and put $\mathfrak{T}_{i}=\left(\mathfrak{E}, c_{i}^{-1} s\right)$, $\Delta_{i}^{*}=\left\{\left(\underline{y}_{i}, J_{i}\right)\right\}$ and $\Delta_{i 0}^{*}=\emptyset$. Since $\Delta_{i}^{*}$ is an EE-regular division of $J_{i}$ and $\Delta_{i 0}^{*}$ is vacuously $c_{i}^{-1} s$-thin, we have $\Delta_{i}^{*} \in J_{i}\left(\mathfrak{T}_{i}\right)$, and hence

$$
-\mathfrak{T}_{i}\left(-f, J_{i}\right) \leq f\left(\underline{y}_{i}\right)\left|J_{i}\right| \leq \mathfrak{T}_{i}\left(f, J_{i}\right)
$$

Also, by Theorem 4.1 (i) $-\mathfrak{T}_{i}\left(-f, J_{i}\right) \leq \mathcal{S}\left(f, J_{i}\right) \leq \mathfrak{T}_{i}\left(f, J_{i}\right)$. Hence

$$
\left|\mathcal{S}\left(f, J_{i}\right)-f\left(\underline{y}_{i}\right)\right| J_{i}|| \leq \mathfrak{T}_{i}\left(f, J_{i}\right)+\mathfrak{T}_{i}\left(-f, J_{i}\right)=\mathfrak{T}_{i}^{o}\left(f, J_{i}\right),
$$

whence the required result follows at once, recalling Lemma 4.3 (i).

In the second case, since $\sum_{i} s\left(\underline{y}_{i}, J_{i}\right)<1$, we can choose $c_{i}>s\left(\underline{y}_{i}, J_{i}\right)$ such that $\sum_{i} c_{i}<1$. Let now $\mathfrak{T}_{i}=\left(\mathfrak{E}, c_{i}^{-1} s\right), \Delta_{i}^{*}=\emptyset$ and $\Delta_{i 0}^{*}=\left\{\left(y_{i}, J_{i}\right)\right\}$. Then $\Delta_{i}^{*}$ is vacuously $\mathfrak{E}$-regular and $\Delta_{i}^{*}+\Delta_{i 0}^{*}$ is a division of $J_{i}$, where $\Delta_{i 0}^{*}$ is $c_{i}^{-1} s$-thin because $c_{i}^{-1} s\left(\Delta_{i o}^{*}\right)=c_{i}^{-1} s\left(\underline{y}_{i}, J_{i}\right)<1$. So $\Delta_{i}^{*} \in J_{i}\left(\mathfrak{T}_{i}\right)$. Since in this case $-\mathfrak{T}_{i}\left(-f, J_{i}\right) \leq-(-f)\left(\Delta_{i}^{*}\right)=f\left(\Delta_{i}^{*}\right)=0 \leq \mathfrak{T}_{i}\left(f, J_{i}\right)$, it follows as above that $\sum\left|\mathcal{S}\left(f, J_{i}\right)\right| \leq \mathfrak{T}^{o}(f, I)$.

Theorem 4.7. If $f \leq g+c$ a.e. on $I$, for some constant $c \geq 0$, then

$$
\mathcal{S}(f, I) \leq \mathcal{S}(g, I)+c|I|
$$

Consequently if $f=g$ a.e. on $I$, then $\mathcal{S}(f, I)=\mathcal{S}(g, I)$ and $\mathcal{S}(-f, I)=$ $\mathcal{S}(-g, I)$ and hence, if further $g$ is $\mathcal{S}$-integrable on $I$, then $f$ is also and to the same value. In particular if $f=0$ a.e. on $I$, then $f$ is $\mathcal{S}$-integrable on $I$ and $\mathcal{S}(f, I)=0$.

Proof. Let $A=\{\underline{x} ; \underline{x} \in I, f(\underline{x}) \leq g(\underline{x})+c\}, E=I \backslash A, h=f-g-c$, and $\eta>0$. Since $|E|=0$, by Lemma 3.4 , there is a $Y$-form $\mathfrak{E}^{0}$ such that for every $\mathfrak{E}^{0}$-regular division $\Delta_{0}^{*}$ over $E$ we have $h\left(\Delta_{0}^{*}\right)<\eta$. Now, given any $\mathcal{S}$-trimmer $\mathfrak{T}=(\mathfrak{E}, s)$ let $\mathfrak{E}^{1}$ be the $Y$-form which is an enumeration of the family of sets $\left\{E_{i} \backslash E, E_{i} \cap E_{j}^{0} \cap E ; i, j=1, \ldots\right\}$. Then $\mathfrak{T}^{1}=\left(\mathfrak{E}^{1}, s\right)$ is an $\mathcal{S}$-trimmer. Take any $\Delta^{*} \in I\left(\mathfrak{T}^{1}\right)$. By Lemma $3.1 \Delta^{*} \in I(\mathfrak{T})$. Also, $\Delta^{*}=\Delta_{1}^{*}+\Delta_{0}^{*}$ where $\Delta_{1}^{*}$ is over $A$ and $\Delta_{0}^{*}$ is over $E$. Besides, since $Y \backslash E$ and $E$ are disjoint, and $\Delta_{0}^{*}$ is $\mathfrak{E}^{1}$-regular, clearly $\Delta_{0}^{*}$ is $\mathfrak{E}^{0}$-regular. Then noting that $h \leq 0$ on $A$ we have

$$
\begin{aligned}
f\left(\Delta^{*}\right) & =(g+c+h)\left(\Delta^{*}\right)=g\left(\Delta^{*}\right)+c|\Delta|+h\left(\Delta^{*}\right) \\
& \leq \mathfrak{T}(g, I)+c|I|+h\left(\Delta_{0}^{*}\right)+h\left(\Delta_{1}^{*}\right) \\
& \leq \mathfrak{T}(g, I)+c|I|+\eta, \Delta^{*} \in I\left(\mathfrak{T}^{1}\right) .
\end{aligned}
$$

So $\mathcal{S}(f, I) \leq \mathfrak{T}^{1}(f, I) \leq \mathfrak{T}(g, I)+c|I|+\eta$, whence by the arbitrariness of $\mathfrak{T}$ and $\eta$ we get (9). 
Remark 4.8. In view of Theorem 4.7, we could speak of $\mathcal{S}$-integrability of functions defined and real valued a.e. on I. Besides, using the technique of McShane [16], the interval I could be replaced by any set in $Y$.

The last part of Theorem 4.7 has the following useful converse.

Theorem 4.9. If $(\mathcal{S}) \int_{J} f=0$ for all $J$ in $I$, then $f=0$ a.e. on $I$.

Proof. It is enough to show that for every $\eta>0,|E| \leq 2 \eta$ where $E=\{y ; \underline{y} \in$ $I,|f(\underline{y})| \geq \eta\}$. Now, by Theorem 4.1 (ii) there is an $\mathcal{S}$-trimmer $\mathfrak{T}=(\mathfrak{E}, s)$ such that $\widetilde{T}^{o}(f, I)<\eta^{2}$. Let $E^{n}=\bigcup_{j=1}^{n}\left(E_{j} \cap E\right)$, and let $\mathfrak{T}^{1}=\left(\mathfrak{E}, s_{1}\right)$ where $s_{1}(\underline{y}, J)=\eta^{-1}|J|$. By Lemma 3.2, for each $n$ there is a $\mathfrak{T}^{1}$-division $\Delta_{n}^{*}$ about $E^{n}$ in $I$, with $\Delta_{n}^{*} \subseteq \Delta_{n+1}^{*}$. Then $\Delta_{n}^{*}=\Delta_{n 1}^{*}+\Delta_{n 0}^{*}$, where $\Delta_{n 1}^{*}$ is E-regular and $s_{1}\left(\Delta_{n 0}^{*}\right)<1$, that is $\left|\Delta_{n 0}\right|<\eta$.

Write $\Delta_{n 1}^{*}=\left\{\left(\underline{y}_{i}, J_{i}\right)\right\}, n$ fixed. Since $\underline{y}_{i} \in E^{n} \subseteq E,\left|f\left(\underline{y}_{i}\right)\right| \geq \eta$ and by hypothesis $\mathcal{S}\left(f, J_{i}\right) \stackrel{2}{=}$. Hence using Lemma 4.6 we get

$$
\left|\Delta_{n 1}\right|=\sum\left|J_{i}\right| \leq \frac{1}{\eta} \sum\left|0-f\left(\underline{y}_{i}\right)\right| J_{i}|| \leq \frac{1}{\eta} \mathfrak{T}^{o}(f, I)<\eta
$$

Thus $\left|\Delta_{n}\right|=\left|\Delta_{n 1}\right|+\left|\Delta_{n 0}\right|<2 \eta$ for all $n$. Since $\Delta_{n} \subseteq \Delta_{n+1}$ and $\bigcup_{n=1}^{\infty} \Delta_{n}$ covers $E$, we get that $|E| \leq 2 \eta$.

Our next theorem is the best possible of its type.

Theorem 4.10. Let $h=\min \{f, g\}$. If $f$ and $g$ are $\mathcal{S}$-integrable on $I$ and $\mathcal{S}(h, I)>-\infty$, then $h$ is $\mathcal{S}$-integrable on $I$.

Proof. By Theorem 4.7, $\mathcal{S}(h, J) \leq \mathcal{S}(f, J)$ and $\mathcal{S}(h, J) \leq \mathcal{S}(g, J)$ for all $J$ in $I$. In particular $-\infty<\mathcal{S}(h, I) \leq \mathcal{S}(f, I)<\infty$. Now, given $\eta>0$, by (FDP) there is an $\mathcal{S}$-trimmer $\mathfrak{T}=(\mathfrak{E}, s)$ such that $\mathfrak{T}^{o}(f, I)<\eta / 3$ and $\mathfrak{T}^{o}(g, I)<\eta / 3$. By definition, every $\Delta^{*} \in I(\mathfrak{T})$ is $\mathfrak{E}$-regular and it can be extended to a division $\Delta^{*}+\Delta_{0}^{*}$ of $I$ such that $\Delta_{0}^{*}$ is $s$-thin. Let $\Delta^{*}=\left\{\left(\underline{y}_{i}, J_{i}\right)\right\}$ and $\Delta_{0}^{*}=\left\{\left(\underline{x}_{j}, I_{j}\right)\right\}$. Then $\left\{J_{i}, I_{j}\right\}$ is a subdivision of $I$. So, using Theorem 4.4 for $h$ and Lemma 
4.6 for $f$ and $g$, we get on writing $A=\left\{i ; h\left(\underline{y}_{i}\right)=f\left(\underline{y}_{i}\right)\right\}$,

$$
\begin{aligned}
&(-h)\left(\Delta^{*}\right)= \sum_{i \in A}\left[\mathcal{S}\left(h, J_{i}\right)-h\left(\underline{y}_{i}\right)\left|J_{i}\right|\right]+\sum_{j} \mathcal{S}\left(h, I_{j}\right) \\
&+\sum_{i \notin A}\left[\mathcal{S}\left(h, J_{i}\right)-h\left(\underline{y}_{i}\right)\left|J_{i}\right|\right]-\mathcal{S}(h, I) \\
& \leq \sum_{i \in A}\left[\mathcal{S}\left(f, J_{i}\right)-f\left(\underline{y}_{i}\right)\left|J_{i}\right|\right]+\sum_{j} \mathcal{S}\left(f, I_{j}\right) \\
&+\sum_{i \notin A}\left[\mathcal{S}\left(g, J_{i}\right)-g\left(\underline{y}_{i}\right)\left|J_{i}\right|\right]-\mathcal{S}(h, I) \\
& \leq \mathfrak{T}^{o}(f, I)+\mathfrak{T}^{o}(f, I)+\mathfrak{T}^{o}(g, I)-\mathcal{S}(h, I) \\
&< \eta-\mathcal{S}(h, I), \Delta^{*} \in I(\mathfrak{T}) .
\end{aligned}
$$

Hence $\mathcal{S}(-h, I) \leq \mathfrak{T}(-h, I) \leq \eta-\mathcal{S}(h, I)$, which by Theorem 4.1 (i) gives $-\mathcal{S}(h, I) \leq \mathcal{S}(-h, I) \leq \eta-\mathcal{S}(h, I)$. Letting $\eta$ tend to zero, we get $-\mathcal{S}(-h, I)=$ $\mathcal{S}(h, I) \neq \pm \infty$ as required.

Theorem 4.11. Let $f$ be $\mathcal{S}$-integrable on I. If $\mathcal{S}(-|f|, I)>-\infty$, in particular if $f$ is essentially bounded above or below in $I$, then $|f|$ is $\mathcal{S}$-integrable on $I$ and $|\mathcal{S}(f, I)| \leq \mathcal{S}(|f|, I)$.

Proof. Since $-|f|=\min \{f,-f\}$, by Theorem 4.10 and Theorem $4.2|f|$ is $\mathcal{S}$-integrable on $I$ and the inequality is obvious.

If $f$ is essentially bounded above on $I$, take an $n$ such that $f(\underline{x})<n$ a.e. on $I$. Then $f(\underline{x})<2 n-|f(\underline{x})|$ a.e. on $I$ and so by Theorem 4.7

$$
-\infty<\mathcal{S}(f, I) \leq \mathcal{S}(-|f|, I)+2 n|I| .
$$

If $f$ is essentially bounded below, then $-f$ is essentially bounded above and so $-\infty<\mathcal{S}(-|-f|, I)=\mathcal{S}(-|f|, I)$.

Notation In the sequel, we shall write $F=\mathcal{S}(f: I)$ to mean that $f$ is $\mathcal{S}$ integrable on $I$ and that $F$ is the indefinite $\mathcal{S}$-integral, or $\mathcal{S}$-primitive of $f$ on $I$, namely the interval function defined for all $J$ in $I$ by $F(J)=\mathcal{S}(f, J)$; recall Theorem 4.5.

\section{Convergence Theorems}

In this section we present two new types of convergence theorems: Singular and Uniform, for sequences of $\mathcal{S}$-integrable functions, in the form of necessary and sufficient conditions for convergence of both the integrands and the integrals. 
From these we then quickly obtain the monotone convergence theorem, Fatou's lemma and the dominated convergence theorem.

The singular convergence theorem has the widest possible range of application. The uniform convergence theorem is restricted precisely by the uniform convergence of the sequence of primitives, and as such it supersedes both the controlled convergence theorem and the generalized mean convergence theorem of Lee, $[15,7.6$, p. 39; 9.7, p. 55] and also compare [1, 10, 11, 23].

Lemma 5.1. Let $F_{n}=\mathcal{S}\left(f_{n}: I\right), n=1, \ldots$, let $p(y)$ be a positive integer for each $y$ in $I$, and let $\eta>0$. Then there is a $Y$-form $\mathfrak{E}$, such that for every $\mathfrak{E}$-regular division $\left\{\left(\underline{y}_{i}, J_{i}\right)\right\}$ in I we have $\sum_{i}\left|F_{p\left(\underline{y}_{i}\right)}\left(J_{i}\right)-f_{p\left(\underline{y}_{i}\right)}\left(\underline{y}_{i}\right)\right| J_{i}||<\eta$.

Proof. By Theorem 4.1 (ii), there are $\mathcal{S}$-trimmers $\mathfrak{T}^{j}=\left(\mathfrak{E}^{j}, s_{j}\right)$ such that $\left(\mathfrak{T}^{j}\right)^{o}\left(f_{j}, I\right)<2^{-j} \eta, j=1, \ldots$ Let $A_{j}=\{y ; y \in I, p(\underline{y})=j\}$. $\left\{A_{j}\right\}$ is a disjoint $I$-form. Then let $\mathfrak{E}$ be the $Y$-form which is an enumeration of the family of sets $\left\{Y \backslash I, A_{j} \cap E_{k}^{j} ; j, k=1, \ldots\right\}$. Now, for any $\mathfrak{E}$-regular $(y, J)$ in $I$ there is a unique $j$ such that $y \in A_{j}$, and then $(y, J)$ is clearly $\mathfrak{E}^{j}$-regular. Hence, by Lemma 4.6, for every $\mathfrak{E}$-regular division $\left\{\left(\underline{y}_{i}, J_{i}\right)\right\}$ in $I$ we have

$$
\begin{aligned}
\sum_{i}\left|F_{p\left(\underline{y}_{i}\right)}\left(J_{i}\right)-f_{p\left(\underline{y}_{i}\right)}\left(\underline{y}_{i}\right)\right| J_{i}|| & =\sum_{j} \sum_{\underline{y}_{i} \in A_{j}}\left|F_{j}\left(J_{i}\right)-f_{j}\left(\underline{y}_{i}\right)\right| J_{i}|| \\
& \leq \sum_{j}\left(\mathfrak{T}^{j}\right)^{o}\left(f_{j}, I\right)<\sum_{j} \frac{\eta}{2^{j}}=\eta .
\end{aligned}
$$

A sequence of functions $\left\{f_{n}\right\}$ is said to be $\mathcal{S}$-integrally, [uniformly $\mathcal{S}$ integrally], Cauchy on $I$ if each $f_{n}$ is $\mathcal{S}$-integrable on $I$, with $F_{n}=\mathcal{S}\left(f_{n}: I\right)$ say, and if for each $\eta>0$ there are positive integers $m$ and $N(\underline{y}), \underline{y} \in I$, such that for every choice of integers $k \geq m$ and $p(y) \geq N(y), y \in I$, there is an $\mathcal{S}$-trimmer $\mathfrak{T}$ such that for every $\mathfrak{T}$-regular division $\left\{\left(\underline{y}_{i}, \overline{J_{i}}\right)\right\}$ for $I$, [for any subinterval $J$ of $I]$, we have

$C(\eta): \quad\left|\sum_{i}\left(f_{p\left(\underline{y}_{i}\right)}-f_{k}\right)\left(\underline{y}_{i}\right)\right| J_{i}||<\eta$.

The $\{m, N(\underline{y})\}$ and $\mathfrak{T}$ are then said to correspond to $\left\{f_{n}\right\}$ and $\{k, p(\underline{y})\}$, respectively, on $I$, [uniformly], in the $C(\eta)$ sense.

Lemma 5.2. Let $F_{n}=\mathcal{S}\left(f_{n}: I\right), n=1, \ldots$ Then $\left\{f_{n}\right\}$ is $\mathcal{S}$-integrally, [uniformly $\mathcal{S}$-integrally], Cauchy on I if and only if for each $\eta>0$ there are positive integers $m$ and $N(\underline{y}), \underline{y} \in I$, such that for every choice of integers 
$k \geq m$ and $p(y) \geq N(y), y \in I$, there is an $\mathcal{S}$-trimmer $\mathfrak{T}$ such that for every T-regular division $\left\{\left(\underline{y}_{i}, J_{i}\right)\right\}$ for $I$, [for any subinterval $J$ of $I$ ], we have

$C^{\prime}(\eta): \quad\left|\sum_{i}\left(F_{p\left(\underline{y}_{i}\right)}-F_{k}\right)\left(J_{i}\right)\right|<\eta$.

Proof. Since $F_{n}-F_{k}=\mathcal{S}\left(f_{n}-f_{k}: I\right)$ for all $n$, each part of the lemma follows readily by applying Lemma 5.1 to the sequence $\left\{f_{n}-f_{k}\right\}_{n=1}^{\infty}$ and using (FDP) in the obvious way, with $\eta / 2$ in place of $\eta$ and without altering $\{m, N(\underline{y})\}$.

Note 5.3. Since the condition $C^{\prime}(\eta)$ involves only $\mathcal{S}$-integrals, by Theorem 4.7 the values of the functions $f_{n}$ can be altered on a set of measure zero both for the application and for the verification of the conditions $C(\eta)$ and $C^{\prime}(\eta)$.

Lemma 5.4. If $\left\{f_{n}\right\}$ is $\mathcal{S}$-integrally Cauchy on $I$, then $\left\{\mathcal{S}\left(f_{n}, I\right)\right\}$ is convergent and $\left\{f_{n}\right\}$ is convergent pointwise a.e.

Proof. Let $F_{n}=\mathcal{S}\left(f_{n}: I\right)$. Given $\eta>0$, let $\{m, N(\underline{y})\}$ correspond to $\left\{f_{n}\right\}$ on $I$ in the $C(\eta)$ sense. Choose arbitrary integers $j>m$ and $k>m$ and let $p(y)=N(y)$ for all $y$ in $I$. Then by (FDP) there is an $\mathcal{S}$-trimmer $\mathfrak{T}$ which corresponds to both $\{j, p(\underline{y})\}$ and $\{k, p(\underline{y})\}$ on $I$ in the $C(\eta)$ sense, and also satisfies $\mathfrak{T}^{o}\left(f_{j}-f_{k}, I\right)<\eta$. Then taking a $\mathfrak{T}$-regular division $\left\{\left(\underline{y}_{i}, J_{i}\right)\right\}$ for $I$, and using Theorem 4.1 (i) and $C(\eta)$, we have

$$
\begin{aligned}
\left|F_{j}(I)-F_{k}(I)\right| \leq & \left|\left(F_{j}-F_{k}\right)(I)-\sum_{i}\left(f_{j}-f_{k}\right)\left(\underline{y}_{i}\right)\right| J_{i}|| \\
& +\left|\sum_{i}\left(f_{j}-f_{p\left(\underline{y}_{i}\right)}\right)\left(\underline{y}_{i}\right)\right| J_{i}||+\left|\sum_{i}\left(f_{p\left(\underline{y}_{i}\right)}-f_{k}\right)\left(\underline{y}_{i}\right)\right| J_{i}|| \\
< & \mathfrak{T}^{o}\left(f_{j}-f_{k}, I\right)+\eta+\eta<3 \eta .
\end{aligned}
$$

Thus the sequence $\left\{F_{n}(I)=\mathcal{S}\left(f_{n}, I\right)\right\}$ is Cauchy and so convergent.

The second part will be proved if we can show that, for any $\eta>0$, if $E$ is the set of points $y$ in $I$ for which there are arbitrarily large integers $p, q$ satisfying $\left|f_{p}(\underline{y})-\overline{f_{q}}(\underline{y})\right| \geq \eta$, then $|E| \leq 3 \eta$. Let $\{m, N(\underline{y})\}$ correspond to $\left\{f_{n}\right\}$ on $I$ in the $C\left(\eta^{2}\right)$ sense. For each $\underline{y}$ in $E$ we choose $p(\underline{y})>N(\underline{y})$ and $q(\underline{y})>N(\underline{y})$ such that

$$
f_{p(\underline{y})}(\underline{y})-f_{q(\underline{y})}(\underline{y}) \geq \eta,
$$

and for each $\underline{y}$ in $I \backslash E$ we choose $p(\underline{y})=q(\underline{y})=N(\underline{y})$. Then, by (FDP), there is an $\mathcal{S}$-trimmer $\mathfrak{T}=(\mathfrak{E}, s)$ which corresponds to both $\{m, p(\underline{y})\}$ and to $\{m, q(\underline{y})\}$ on $I$ in the $C\left(\eta^{2}\right)$ sense. 
Now let $E^{n}=\bigcup_{j=1}^{n}\left(E_{j} \cap E\right)$, and let $\mathfrak{T}^{1}=\left(\mathfrak{E}, s_{1}\right)$ where $s_{1}(\underline{y}, J)=|J| / \eta$. By Lemma 3.2, for each $n$ there is a $\mathfrak{T}^{1}$-division $\Delta_{n}^{*}$ about $E^{n}$ in $I$, with $\Delta_{n}^{*} \subseteq \Delta_{n+1}^{*}$. Then $\Delta_{n}^{*}=\Delta_{n 1}^{*}+\Delta_{n 0}^{*}$ where $\Delta_{n 1}^{*}$ is $\mathfrak{E}$-regular and $s_{1}\left(\Delta_{n 0}^{*}\right)<1$, that is $\left|\Delta_{n 0}\right|<\eta$.

Fix any $n$. As in the proof of Lemma 3.2, we can extend $\Delta_{n 1}^{*}$ to a T-regular division $\left\{\left(y_{i}, J_{i}\right)\right\}$ for $I$. Then, noting that $\Delta_{n 1}^{*}$ is over $E$ and $p\left(\underline{y}_{i}\right)=q\left(\underline{y}_{i}\right)$ for all $\underline{y}_{i} \notin E$, by (10) and $C\left(\eta^{2}\right)$ we have

$$
\begin{aligned}
\eta\left|\Delta_{n 1}\right| & \leq \eta \sum_{\underline{y}_{i} \in E}\left|J_{i}\right| \leq \sum_{\underline{y}_{i} \in E}\left(f_{p\left(\underline{y}_{i}\right)}-f_{q\left(\underline{y}_{i}\right)}\right)\left(\underline{y}_{i}\right)\left|J_{i}\right| \\
& =\sum_{i}\left(f_{p\left(\underline{y}_{i}\right)}-f_{m}\right)\left(\underline{y}_{i}\right)\left|J_{i}\right|+\sum_{i}\left(f_{m}-f_{q\left(\underline{y}_{i}\right)}\right)\left(\underline{y}_{i}\right)\left|J_{i}\right| \\
& <\eta^{2}+\eta^{2}=2 \eta^{2} .
\end{aligned}
$$

Thus $\left|\Delta_{n}\right|=\left|\Delta_{n 1}\right|+\left|\Delta_{n 0}\right|<2 \eta+\eta$ for all $n$. Since $\Delta_{n} \subseteq \Delta_{n+1}$ and $\cup_{n=1}^{\infty} \Delta_{n}$ covers $E$, we get $|E| \leq 3 \eta$.

Theorem 5.5. (Singular Convergence) Let $F_{n}=\mathcal{S}\left(f_{n}: I\right), n=1, \ldots$. The following conditions are equivalent.

(i) The sequence $\left\{f_{n}\right\}$ is $\mathcal{S}$-integrally Cauchy on I.

(ii) $\left\{f_{n}\right\}$ converges pointwise a.e. to an $\mathcal{S}$-integrable function $f_{0}$ on $I$, and also $\left\{\mathcal{S}\left(f_{n}, I\right)\right\}$ converges to $c=\mathcal{S}\left(f_{0}, I\right)$.

Proof. Assume (i). By Lemma $5.4\left\{f_{n}\right\}$ converges pointwise a.e. to a function $f_{0}$ on $I$, and $\left\{\mathcal{S}\left(f_{n}, I\right)=F_{n}(I)\right\}$ converges to a number $c$. For the integrals in (ii), by Note 5.3 , we can assume further that $\left\{f_{n}\right\}$ converges everywhere to $f_{0}$ on $I$. Now, given $\eta>0$, let $\{m, N(y)\}$ correspond to $\left\{f_{n}\right\}$ on $I$ in the $C(\eta)$ sense. We choose integers $p(\underline{y})>N(\underline{y}), \underline{y} \in I$, and $k>m$ such that

$$
\left|f_{0}(\underline{y})-f_{p(\underline{y})}(\underline{y})\right|<\eta \text { and }\left|F_{k}(I)-c\right|<\eta \text {. }
$$

Then, by (FDP), there is an $\mathcal{S}$-trimmer $\mathfrak{T}$ which corresponds to $\{k, p(\underline{y})\}$ on $I$ in the $C(\eta)$ sense, and also satisfies $\mathfrak{T}^{o}\left(f_{k}, I\right)<\eta$. Then for all $\Delta^{*} \in \mathcal{\in} I(\mathfrak{T})$, 
$\Delta^{*}=\left\{\left(\underline{y}_{i}, J_{i}\right)\right\}$, using $C(\eta)$ and Theorem 4.1 (i) we have

$$
\begin{aligned}
\left|f_{0}\left(\Delta^{*}\right)-c\right| \leq & \sum_{i}\left|\left(f_{0}-f_{p\left(\underline{y}_{i}\right)}\right)\left(\underline{y}_{i}\right)\right|\left|J_{i}\right| \\
& +\left|\sum_{i}\left(f_{p\left(\underline{y}_{i}\right)}-f_{k}\right)\left(\underline{y}_{i}\right)\right| J_{i}|| \\
& +\left|\sum_{i} f_{k}\left(\underline{y}_{i}\right)\right| J_{i}\left|-F_{k}(I)\right|+\left|F_{k}(I)-c\right| \\
< & \sum_{i} \eta\left|J_{i}\right|+\eta+\mathfrak{T}^{o}\left(f_{k}, I\right)+\eta<\eta(|I|+3) .
\end{aligned}
$$

Hence, by Theorem 4.1 (iii) we have $(\mathcal{S}) \int_{I} f_{o}=c$, proving (ii).

Conversely, assume (ii). To prove (i), by Note 5.3 we can assume further that $\left\{f_{n}\right\}$ converges to $f_{0}$ everywhere on $I$. Then given an $\eta>0$, there are positive integers $m$ and $N(\underline{y}), \underline{y} \in I$ such that for every choice of integers $k \geq m$ and $p(\underline{y}) \geq N(\underline{y}), \underline{y} \in I$ we have

$$
\left|f_{p(\underline{y})}-f_{0}\right|(\underline{y})<\tau=\frac{\eta}{|I|+3} \text { and }\left|c-F_{k}(I)\right|<\tau .
$$

Also, by (FDP), there is an $\mathcal{S}$-trimmer $\mathfrak{T}$ such that $\mathfrak{T}^{o}\left(f_{j}, I\right)<\tau, j=0, k$. Then for all $\left\{\left(\underline{y}_{i}, J_{i}\right)\right\} \in I(\mathfrak{T})$, we have

$$
\begin{aligned}
\left|\sum_{i}\left(f_{p\left(\underline{y}_{i}\right)}-f_{k}\right)\left(\underline{y}_{i}\right)\right| J_{i}|| \leq\left|\sum_{i}\left(f_{p\left(\underline{y}_{i}\right)}-f_{0}\right)\left(\underline{y}_{i}\right)\right| J_{i}|| \\
\quad+\left|\sum_{i} f_{0}\left(\underline{y}_{i}\right)\right| J_{i}|-c|+\left|c-F_{k}(I)\right|+\left|F_{k}(I)-\sum_{i} f_{k}\left(\underline{y}_{i}\right)\right| J_{i}|| \\
\quad<\sum_{i} \tau\left|J_{i}\right|+\mathfrak{T}^{o}\left(f_{0}, I\right)+\tau+\mathfrak{T}^{o}\left(f_{k}, I\right)<\tau|I|+3 \tau=\eta .
\end{aligned}
$$

Thus $\{m, N(y)\}$ corresponds to $\left\{f_{n}\right\}$ on $I$ in the $C(\eta)$ sense. Hence $\left\{f_{n}\right\}$ is $\mathcal{S}$-integrally Cauchy on $I$, proving (i).

Theorem 5.6. (Uniform Convergence) Let $F_{n}=\mathcal{S}\left(f_{n}: I\right), n=1, \ldots$. The following conditions are equivalent.

(i) The sequence $\left\{f_{n}\right\}$ is uniformly $\mathcal{S}$-integrally Cauchy on $I$.

(ii) $\left\{f_{n}\right\}$ converges pointwise a.e. to a $\mathcal{S}$-integrable function $f_{0}$ on $I$ and also $\left\{F_{n}\right\}$ converges uniformly on $I$ to $F_{0}=\mathcal{S}\left(f_{0}: I\right)$, that is $F_{n}(J) \rightarrow F_{0}(J)$ uniformly for all $J$ in $I$. 
Proof. Assume (i). Then obviously, $\left\{f_{n}\right\}$ is $\mathcal{S}$-integrally Cauchy on every subinterval $J$ of $I$. So obviously by Theorem $5.5,\left\{f_{n}\right\}$ converges pointwise a.e. to an $\mathcal{S}$-integrable function $f_{0}$ on $I$. Writing $F_{0}=\mathcal{S}\left(f_{0}: I\right)$, we also have that $F_{n}(J) \rightarrow F_{0}(J)$, for all $J$ in $I$. Now, given $\eta>0$, let $\{m, N(y)\}$ correspond to $\left\{f_{n}\right\}$ uniformly on $I$ in the $C(\eta)$ sense. Choose arbitrary integers $j>m$ and $k>m$, and let $p(\underline{y})=N(\underline{y})$ for all $\underline{y}$ in $I$. Then, recalling Lemma 4.3 (ii), by (FDP) there is an $\mathcal{S}$-trimmer $\mathfrak{T}=(\mathfrak{E}, s)$ which corresponds to both $\{j, p(y)\}$ and $\{k, p(y)\}$ uniformly on $I$ in the $C(\eta)$ sense, and also satisfies $\mathfrak{T}^{o}\left(f_{j}^{-}-f_{k}, J\right)<\eta$ for all $J$ in $I$. Then, for all $J$ in $I$, taking a $\left\{\left(y_{i}, J_{i}\right)\right\} \in J(\mathfrak{T})$ it follows that as in the first part of the proof of Lemma 5.4, with $J$ for $I$, that $\left|F_{j}(J)-F_{k}(J)\right|<3 \eta$. Hence $\left\{F_{n}\right\}$ converges uniformly to $F_{0}$ on $I$, proving (ii).

Conversely, assume (ii). To prove (i), by Note 5.3 we can assume further that $\left\{f_{n}\right\}$ converges to $f_{0}$ everywhere on $I$. Then, given $\eta>0$, there are positive integers $m$ and $N(\underline{y}), \underline{y} \in I$, such that for every choice of integers $k \geq m$ and $p(\underline{y}) \geq N(\underline{y}), \underline{y} \in I$, and for all $J$ in $I$, we have

$$
\left|f_{p(\underline{y})}-f_{0}\right|(\underline{y})<\tau=\frac{\eta}{|I|+3} \text { and }\left|F_{0}(J)-F_{k}(J)\right|<\tau .
$$

Also, recalling Lemma 4.3 (ii), by (FDP) there is an $\mathcal{S}$-trimmer $\mathfrak{T}$ such that for $j=0, k, \mathfrak{T}^{o}\left(f_{j}, J\right)<\tau$ for all $J$ in $I$. Then considering all $J$ in $I$, and all $\left\{\left(y_{i}, J_{i}\right)\right\} \in J(\mathfrak{T})$ it follows that as in the last part of the proof of Theorem 5.5 , with $J$ for $I$ and $F_{0}(J)$ for $c$, that $\mathfrak{T}$ corresponds to $\{k, p(y)\}$ uniformly on $I$ in the $C(\eta)$ sense, proving (i).

Remark 5.7. In contrast to uniform convergence, singular convergence is extremely pathological. For example, in $\mathbb{R}$, let

$$
f_{n}(x)= \begin{cases}n & \text { if } 0<x<\frac{1}{n} \\ -n & \text { if } 3-\frac{1}{n}<x<3 \\ 0 & \text { otherwise. }\end{cases}
$$

Then $\left\{f_{n}\right\}$ converges to $f_{0}=0$ everywhere. Assuming that $\mathcal{S}$ in $\mathbb{R}$ contains the length sifter $s, s(y, J)=|J|$. It is easy to verify that $(\mathcal{S}) \int_{I} f_{n}=0$ where $I=[0,3]$, and so Theorem 5.5 holds trivially. But, on the other hand, for the subinterval $J=[0,1],(\mathcal{S}) \int_{J} f_{n}=1$, while $(\mathcal{S}) \int_{J} f_{0}=0$.

Theorem 5.8. (Monotone Convergence) Let $F_{n}=\mathcal{S}\left(f_{n}: I\right), n=1, \ldots$, let $f_{n} \leq f_{n+1},\left[f_{n} \geq f_{n+1}\right]$, a.e. on $I$ for each $n$ and let $\sup _{n} F_{n}(I)<\infty$, $\left[\inf _{n} F_{n}(I)>-\infty\right]$. Then $\left\{f_{n}\right\}$ converges pointwise a.e. to an $\mathcal{S}$-integrable function $f_{0}$ on $I$, and also $\left\{F_{n}\right\}$ converges uniformly to $F_{0}=\mathcal{S}\left(f_{0}: I\right)$ on $I$. 
Proof. Looking at $\left\{-f_{n}\right\}$, we prove the first case only. Obviously, the sequence $\left\{F_{n}(I)\right\}$ is nondecreasing and convergent. So, given $\eta>0$, there is a positive integer $m$ such that $0 \leq F_{q}(I)-F_{m}(I)<\eta$ for all $q \geq m$. Let $N(\underline{y})=m, \underline{y} \in I$. Then for every choice of integers $k \geq m$ and $p(y) \geq N(y)=$ $m, \underline{y} \in I$, and every division $\left\{\left(\underline{y}_{i}, J_{i}\right)\right\}$ in $I$, setting $q=k+\sum_{i} p\left(\underline{y}_{i}\right)$, for all $i$ we have

$$
-\left(f_{q}-f_{m}\right) \leq f_{p\left(\underline{y}_{i}\right)}-f_{k} \leq\left(f_{q}-f_{m}\right) \text { a.e. on } I,
$$

and hence $\left|\mathcal{S}\left(f_{p\left(\underline{y}_{i}\right)}-f_{k}, J_{i}\right)\right| \leq \mathcal{S}\left(f_{q}-f_{m}, J_{i}\right)$. Hence

$$
\begin{aligned}
\left|\sum_{i}\left(F_{p\left(\underline{y}_{i}\right)}-F_{k}\right)\left(J_{i}\right)\right| & \leq \sum_{i}\left|\mathcal{S}\left(f_{p\left(\underline{y}_{i}\right)}-f_{k}, J_{i}\right)\right| \leq \sum_{i} \mathcal{S}\left(f_{q}-f_{m}, J_{i}\right) \\
& \leq \mathcal{S}\left(f_{q}-f_{m}, I\right)=F_{q}(I)-F_{m}(I)<\eta .
\end{aligned}
$$

Thus $\{m, N(y)=m\}$ corresponds to $\left\{f_{n}\right\}$ uniformly on $I$ in the $C^{\prime}(\eta)$ sense as in Lemma 5.2 , in a trivial way. Hence by Lemma $5.2,\left\{f_{n}\right\}$ is uniformly $\mathcal{S}$ integrally Cauchy on $I$, in a remarkably strong sense indeed, which by Theorem 5.6 completes the proof.

Lemma 5.9. Let $F_{n}=\mathcal{S}\left(f_{n}: I\right), n=1, \ldots$. If $f_{n} \geq g$ a.e. on I for each $n$, and if $\mathcal{S}(g, I)>-\infty$, then $\inf _{n} f_{n}$ equals a.e. an $\mathcal{S}$-integrable function on $I$.

Proof. Let $h_{n}=\min \left\{f_{1}, \ldots, f_{n}\right\}, n=1, \ldots$ Then $h_{n} \geq g$ a.e. on $I$, and so by Theorem $4.7 \mathcal{S}\left(h_{n}, I\right) \geq \mathcal{S}(g, I)>-\infty$ for all $n$. So from Theorem 4.10 it follows by induction that each $h_{n}$ is $\mathcal{S}$-integrable on $I$. Also, we have $h_{n} \geq h_{n+1}$ on $I$ for all $n$ and $\inf _{n} \mathcal{S}\left(h_{n}, I\right) \geq \mathcal{S}(g, I)>-\infty$. Hence by Theorem 5.8, $\left\{h_{n}\right\}$ converges a.e. to an $\mathcal{S}$-integrable function $h$ on $I$, and the proof ends by noting that $\inf _{n} f_{n}(\underline{x})=\lim _{n} h_{n}(\underline{x})=h(\underline{x})$ a.e. on $I$.

Theorem 5.10. (Fatou's Lemma) Let $F_{n}=\mathcal{S}\left(f_{n}: I\right), n=1, \ldots$.

(i) If $f_{n} \geq g$ a.e. on I for each $n$, and if $\mathcal{S}(g, I)>-\infty$ and $\liminf _{n} F_{n}(I)<$ $\infty$, then $\liminf _{n} f_{n}$ equals a.e. an $\mathcal{S}$-integrable function $h$ on $I$, and further $\mathcal{S}(h, I) \leq \liminf _{n} F_{n}(I)$.

(ii) If $f_{n} \leq g$ a.e. on I for each $n$, and if $\mathcal{S}(-g, I)>-\infty$ and $\lim \sup _{n} F_{n}(I)$ $>-\infty$, then $\lim _{\sup _{n}} f_{n}$ equals a.e. an $\mathcal{S}$-integrable function $h$ on $I$, and further $\mathcal{S}(h, I) \geq \lim \sup _{n} F_{n}(I)$.

Proof. As before, looking at $\left\{-f_{n}\right\}$ we prove only (i).

By Lemma 5.9, for each $n$ there is an $\mathcal{S}$-integrable function $h_{n}$ on $I$ such that $h_{n}(\underline{x})=\inf \left\{f_{k}(\underline{x}) ; k \geq n\right\}$ a.e. on $I$. Clearly $h_{n} \leq h_{n+1}$ and $h_{n} \leq f_{n}$ 
a.e. on $I$ for each $n$, and so $\mathcal{S}\left(h_{n}, I\right) \leq \mathcal{S}\left(h_{n+1}, I\right)$ and $\mathcal{S}\left(h_{n}, I\right) \leq F_{n}(I)$ for all $n$. Hence

$$
\sup _{n} \mathcal{S}\left(h_{n}, I\right)=\lim _{n} \mathcal{S}\left(h_{n}, I\right) \leq \liminf _{n} F_{n}(I)<\infty,
$$

which by Theorem 5.8 for $\left\{h_{n}\right\}$ evidently proves (i).

Theorem 5.11. (Dominated Convergence) Let $F_{n}=\mathcal{S}\left(f_{n}: I\right), n=1, \ldots$, let $\left\{f_{n}\right\}$ converge to $f_{0}$ a.e. on $I$, and let $g \leq f_{n} \leq f$ a.e. on $I$ for each $n$, where $\mathcal{S}(g, I)>-\infty$ and $\mathcal{S}(-f, I)>-\infty$. Then $f_{0}$ is $\mathcal{S}$-integrable on $I$ and $\lim _{n} F_{n}(J)=\mathcal{S}\left(f_{0}, J\right)$ for all $J$ in $I$.

Proof. We have

$$
-\infty<\mathcal{S}(g, I) \leq F_{n}(I)=-\mathcal{S}\left(-f_{n}, I\right) \leq-\mathcal{S}(-f, I)<\infty
$$

for all $n$. So, $\liminf _{n} F_{n}(I)$ and $\limsup _{n} F_{n}(I)$ are both finite. Therefore, by Theorem 5.10 (i,ii) and Theorem 4.7, clearly $f_{0}$ is $\mathcal{S}$-integrable on $I$, and further,

$$
\limsup _{n} F_{n}(I) \leq \mathcal{S}\left(f_{0}, I\right) \leq \liminf _{n} F_{n}(I) .
$$

Thus $\lim _{n} F_{n}(I)=\mathcal{S}\left(f_{0}, I\right)$. We note also that, since by above both $\mathcal{S}(g, I)$ and $\mathcal{S}(-f, I)$ are finite by Theorem 4.4 (ii) both $\mathcal{S}(g, J)$ and $\mathcal{S}(-f, J)$ are finite for all $J$ in $I$. Hence we can replace $I$ with any $J$ in $I$.

\section{Functions $\mathcal{S}$-AC and MAC, Spectral Derivative}

We now pause to introduce and study two distinct generalizations of absolute continuity, AC, [19, p. 93], and a notion of strong approximate derivative, for interval functions. These will be used in the next section to give a DenjoyLusin type descriptive definition of the $\mathcal{S}$-integral. The use of Vitali's covering theorem, [19, p. 109], and the theory of density of sets, [19, p. 128], seems to be indispensable here.

Take any interval function $F$ known for all $J$ in I . If for each $\eta>0$ there is an $s$ in $\mathcal{S}$ such that $|F(\Delta)|<\eta$ for all $s$-thin divisions $\Delta^{*}$ in $I$, then $F$ is said to be $\mathcal{S}$-AC on $I$.

Next, a subdivision $\Delta_{1}$ is said to be subordinate to a subdivision $\Delta$, written $\Delta_{1}$ sub $\Delta$, if each interval of $\Delta_{1}$ is in some, necessarily unique, interval of $\Delta$.

For $E \subseteq I$, we denote by $M(F, E ; I),[U(F, E ; I)]$, the infimum of all $K \leq \infty$ for which there is an $E$-form $\mathbb{E}$ and a $\delta>0$ such that $F(\Delta)-F\left(\Delta_{1}\right)<$ $K$ for all pairs of $\mathfrak{E}$-regular, possibly empty, subdivisions $\Delta, \Delta_{1}$ in $I$ with 
||$\Delta|-| \Delta_{1}||<\delta$, [and with $\Delta_{1} \operatorname{sub} \Delta$ ]. Clearly $M(F, E ; I) \geq U(F, E ; I) \geq 0$. We call $M(F, E ; I)$ the meager variation of $F$ on $E$ in $I$.

If for each $\eta>0$ there is an $I$-form $\left\{E_{n}\right\}$ such that

$$
\sum_{n=1}^{\infty} M\left(F, E_{n} ; I\right)<\eta,\left[\sum_{n=1}^{\infty} U\left(F, E_{n} ; I\right)<\eta\right]
$$

then $F$ is said to be MAC, [MAC-up], on $I$. Using (FDP), it is readily seen that $M(F, A ; I) \leq M(F, E ; I)$ and $U(F, A ; I) \leq U(F, E ; I)$, if $A \subseteq E$, that the properties $\mathcal{S}$-AC and MAC are hereditary and that a linear combination of any two $\mathcal{S}$-AC functions is again $\mathcal{S}$-AC. For MAC this follows from Theorem 6.9 infra. It is natural to call $F$ MAC-down if $-F$ is MAC-up.

Theorem 6.1. For every $E \subseteq I$ there is a measurable set $A$ such that $E \subseteq$ $A \subseteq I$ and $W(F, E ; I)=W(F, A ; I)$, for $W=M, U$.

Proof. Given a positive integer $k$, by (FDP), there is an $E$-form $\mathfrak{E}$ and a $\delta>0$ such that

$$
F(\Delta)-F\left(\Delta_{1}\right) \leq M(F, E ; I)+\frac{1}{k},\left[F(\Delta)-F\left(\Delta_{1}\right) \leq U(F, E ; I)+\frac{1}{k}\right],
$$

for all pairs of $\mathfrak{E}$-regular subdivisions $\Delta, \Delta_{1}$ in $I$ with ||$\Delta|-| \Delta_{1}||<\delta$, [and $\Delta_{1}$ sub $\Delta$ ]. Invoking star-closure (see $\S 3$ ) the set $A_{k}=\cup_{n=1}^{\infty} E_{n}^{+}$is measurable and $E \subseteq A_{k} \subseteq I$. Since $\mathfrak{E}^{+}$is an $A_{k}$-form and $\mathfrak{E}^{+}$-regular subdivisions are E-regular, we have $W\left(F, A_{k} ; I\right) \leq W(F, E ; I)+1 / k$ for $W=M, U$. It is clearly enough to take $A=\cap_{k=1}^{\infty} A_{k}$.

Now recall that a set $E$ has (outer) density 1 at a point $y$ in $Y$, if for each $\eta>0$, we have $|E \cap J|>(1-\eta)|J|$ for all $(\underline{y}, J)$ in some neighborhood of $\underline{y}$.

For each $\underline{y}$ in $I$, we denote by $\bar{F}_{s p}(\underline{y})$ the infimum of all $K \leq \infty$ for which there is a closed set $A$ having density 1 at $\underline{y}$, such that $F(J) \leq K|J|$ for all $A$ regular $J$ in $I ; J$ need not contain $\underline{y}$; the existence of $A$-regular $J$ in $I$ follows from Lemma 2.5. The phrase 'in $I$ ' is superfluous for $\underline{y}$ in $\stackrel{\circ}{I}$.

We call $\bar{F}_{s p}(\underline{y})$ and $-(\overline{-F})_{s p}(\underline{y})$ respectively the upper and lower spectral derivates of $F$ at $y$ in $I$; and if they are equal, their common value is called the spectral derivative of $F$ at $\underline{y}$ in $I, F_{s p}^{\prime}(\underline{y})$.

Routine arguments give us $-\overline{(-F)}_{s p}(\underline{y}) \leq \bar{F}_{s p}(\underline{y})$, as well as other standard results concerning linear combinations.

Theorem 6.2. The spectral derivate $\bar{F}_{s p}$ is measurable on I. 
Proof. For any $\underline{y}_{0}$ in $\stackrel{\circ}{I}$ and any $c>\bar{F}_{s p}\left(\underline{y}_{0}\right)$, taking a $K$ with $c>K>$ $\bar{F}_{s p}\left(y_{0}\right)$ there is a closed set $A$ having density 1 at $y_{0}$ such that $F(J)<K|J|$ for all $A$-regular $J$. Since $A$ is closed and has density 1 a.e. on itself, we have $c>K \geq \bar{F}_{s p}(\underline{y})$ for almost all $\underline{y}$ in $A$. This evidently implies that $\bar{F}_{s p}$ is measurable on $\bar{I}$.

Lemma 6.3. Let $\bar{F}_{s p}>f$ a.e. on a set $E$ in I. Then, for almost all $y$ in $E$ and for every $\eta>0$, every neighborhood of $\underline{y}$ contains an E-regular $(\underline{y}, J)$ in I (Recall Lemma 2.5.) such that

$$
F(J)>f(\underline{y})|J| \text { and } Q(J)>1-\eta .
$$

Proof. Since $\mathbb{R}$ is separable, it is clearly enough to consider the case when $\bar{F}_{s p}>c>f$ a.e. on $E$, where $c$ is a real number. It is then enough to show that, for each $\eta>0$, if $H$ is the set of points $\underline{y}$ of $E$ such that $F(J) \leq c|J|$ for every $E$-regular $(y, J)$ in $I$ with $d(J)<\eta$ and $Q(J)>1-\eta$, then $|H|=0$. Invoking star-closure again, let $A$ be a closed subset of $H^{+}$with $d(A)<\eta$. For any $A$-regular $J$ in $I$, we have $d(J)<d(A)<\eta$ and $Q(J)>1-d(A)>1-\eta$; also since $A \subseteq H^{+} \subseteq E^{+}$, clearly $J$ intersects $H$ and $J$ is $E$-regular, being $E^{+}$-regular. Then, taking $\underline{y} \in H \cap J$ we see that $(\underline{y}, J)$ is $E$-regular and is in $I, d(J)<\eta$, and $Q(J)>1-\eta$. So, by the definition of $H, F(J) \leq c|J|$. Since $A$ is closed and has density 1 a.e. on $H \cap A$, it follows that $\bar{F}_{s p}(y) \leq c$ a.e. on $H \cap A$. Since $H^{+}$is a countable union of closed sets $A$ with $d(\bar{A})<\eta$ and a set of measure zero, we have $\bar{F}_{s p} \leq c$ a.e. on $H$, which by hypothesis gives $|H|=0$.

Recall that the ordinary upper derivate, [19, p. 106], of $F$ at $y$ in $I, \bar{F}(y)$, is the infimum of all $K \leq \infty$ such that, for each $\tau>0, F(J) \leq K|J|$ for all $(\underline{y}, J)$ in some neighborhood of $\underline{y}$ in $I$ with $Q(J)>\tau$. If $\bar{F}(\underline{y})=-\overline{(-F)}(\underline{y})$, then this common value is the ordinary derivative of $F$ at $\underline{y}$ in $I, F^{\prime}(\underline{y})$.

We define the upper cubical derivate of $F$ at $y$ in $I, C \bar{F}(y)$ to be the infimum of all $K \leq \infty$ for which there is an $\eta>0$ such that $\bar{F}(J) \leq K|J|$ for all $(\underline{y}, J)$ in some neighborhood of $\underline{y}$ in $I$ with $Q(J)>1-\eta$. If $C \bar{F}(\underline{y})=$ $-C \overline{(-F)}(\underline{y})$, then this common value is the cubical derivative of $F$ at $\underline{y}$ in $I$, $C F^{\prime}(\underline{y})$.

Theorem 6.4. We have $\bar{F}_{s p} \leq C \bar{F} \leq \bar{F}$ a.e. on I. [In other words $F_{s p}^{\prime}$ is the most general derivative in the a.e. sense.]

Proof. Clearly $C \bar{F} \leq \bar{F}$ on $I$. If $E=\left\{\underline{y} ; \underline{y} \in I, \bar{F}_{s p}(\underline{y})>C \bar{F}(\underline{y})\right\}$, choose an $f$ such that $\bar{F}_{s p}>f>C \bar{F}$ on $E$. From Lemma 6.3 it follows at once that $C \bar{F} \geq f$ a.e. on $E$ and so $|E|=0$. 
For any set $E$ and any $E$-form $\mathfrak{E}$, by a Vitali $\mathfrak{E}$-cover we shall mean a family of intervals $\zeta$ such that, for each $n$ and for almost all $y$ in $E_{n}$, every neighborhood of $\underline{y}$ contains an $E_{n}$-regular $J \in \zeta$ with $\underline{y} \in J$ and $Q(J)>1 / 2$.

Lemma 6.5. If $\bar{F}_{s p}>c>c_{1}>-\overline{(-F)}_{s p}$ a.e. on a set $E$ in $I$, then ( $c-$ $\left.c_{1}\right)|E| \leq U(F, E ; I)$.

Proof. Let $\zeta,\left[\zeta_{1}\right]$, be the family of all $J$ such that $F(J)>c|J|,[-F(J)>$ $\left.-c_{1}|J|\right]$. Take any $E$-form $\mathfrak{E}$ and any $\delta>0$. Since $\bar{F}_{s p}>c$ and $c_{1}>-\overline{(-F)_{s p}}$ a.e. on $E_{n}$ for each $n$, by Lemma 6.3 both $\zeta$ and $\zeta_{1}$ are Vitali E-covers. Hence, clearly by the Vitali covering theorem, given $0<\eta<\delta / 2$ we can successively find E-regular subdivisions $\Delta \subseteq \zeta$ and $\Delta_{1} \subseteq \zeta_{1}$ in $I$ such that

$$
|E|-\eta<|\Delta|<|E \cap \bigcup \Delta|+\eta, \Delta_{1} s u b \Delta \text {, and }|E \cap \bigcup \Delta|-\left|\Delta_{1}\right|<\eta .
$$

Then $0 \leq|\Delta|-\left|\Delta_{1}\right|<2 \eta<\delta$ and we have

$$
\begin{aligned}
F(\Delta)-F\left(\Delta_{1}\right) & \geq c|\Delta|-c_{1}\left|\Delta_{1}\right|=\left(c-c_{1}\right)|\Delta|+c_{1}\left(|\Delta|-\left|\Delta_{1}\right|\right) \\
& >\left(c-c_{1}\right)|E|-\left(c-c_{1}\right) \eta-\left|c_{1}\right| 2 \eta .
\end{aligned}
$$

This evidently implies the required result.

Theorem 6.6. If $U(F, E ; I)<\infty$, then both $\bar{F}_{s p}$ and $\overline{(-F)}_{s p}$ are finite a.e. on $E$. If $F$ is $M A C$-up on $I, F_{s p}^{\prime}$ exists finitely a.e. on $I$.

Proof. There is an $E$-form $\mathfrak{E}$ and a $\delta>0$, such that

$$
F(\Delta)-F\left(\Delta_{1}\right)<U(F, E ; I)+1<\infty,
$$

for all pairs of E-regular subdivisions $\Delta, \Delta_{1}$ in $I$ with $|\Delta|-\left|\Delta_{1}\right|<\delta$ and $\Delta_{1}$ sub $\Delta$. Now, first take a $J_{0}$ in $I$ with $\left|J_{0}\right|<\delta$. Let $E_{0}=\{\underline{y} ; \underline{y} \in E \cap$ $\left.J_{0}, \bar{F}_{s p}(\underline{y})=\infty\right\}$ and put $\mathfrak{E}^{0}=\mathfrak{E} E_{0}$. Let $\eta>0$ and $\zeta$ be the family of all $J$ such that $F(J)>|J| / \eta$. Since $\bar{F}_{s p}>1 / \eta$ on $E_{n}^{0}$ for each $n$, by Lemma $6.3 \zeta$ is a Vitali $\mathfrak{E}^{0}$-cover. Then, by the Vitali covering theorem, there is an $\mathfrak{E}^{0}$-regular and so $\mathfrak{E}$-regular, subdivision $\Delta \subseteq \zeta$ in $J_{0}$ such that ||$E_{0}|-| \Delta \|<\eta$. Since $|\Delta|-|\emptyset| \leq\left|J_{0}\right|<\delta$, we have

$$
\begin{aligned}
U(F, E ; I)+1 & >F(\Delta)-F(\emptyset) \geq \frac{|\Delta|}{\eta} \\
& =\frac{1}{\eta}\left|E_{0}\right|+\frac{1}{\eta}\left(|\Delta|-\left|E_{0}\right|\right)>\frac{\left|E_{0}\right|}{\eta}-1 .
\end{aligned}
$$

Since $\eta>0$ is arbitrary, it follows that $\left|E_{0}\right|=0$. Taking a subdivision $\left\{J_{i}\right\}$ of $I$ with $\left|J_{i}\right|<\delta$ for each $i$, it therefore follows that $\bar{F}_{s p}<\infty$ a.e. on $E$. 
Next, let $E^{1}=\left\{\underline{y} ; \underline{y} \in E, \overline{(-F)}_{s p}(\underline{y})=\infty\right\}, \mathfrak{E}^{1}=\mathfrak{E} E^{1}$ and $0<\eta<$ $\delta / 2$. By Lemma 2.5 , the family of all $\mathfrak{E}^{1}$-regular intervals is a Vitali $\mathfrak{E}^{1}$ cover. So by the Vitali covering theorem, there is an $\mathfrak{E}^{1}$-regular, so $\mathfrak{E}$-regular, subdivision $\Delta$ in $I$ such that $\left|E^{1}\right|-\eta<|\Delta|<\left|E^{1} \cap \bigcup \Delta\right|+\eta$. Take a positive integer $k>(U(F, E ; I)+1-F(\Delta)) / \eta$. Let $\zeta_{1}$ be the family of all $J$ such that $-F(J)>k|J|$. Since $\overline{(-F)}_{s p}>k$ on $E_{n}^{1}$ for each $n$, by Lemma $6.3 \zeta_{1}$ is a Vitali $\mathfrak{E}^{1}$-cover. Hence, clearly by the Vitali covering theorem, there is an $\mathfrak{E}^{1}$-regular, and so $\mathfrak{E}$-regular, subdivision $\Delta_{1} \subseteq \zeta_{1}$ in $I$ such that $\left|E^{1} \cap \bigcup \Delta\right|-\left|\Delta_{1}\right|<\eta$ and $\Delta_{1} s u b \Delta$. Noting that $|\Delta|-\left|\Delta_{1}\right|<2 \eta<\delta$ we have $U(F, E ; I)+1>F(\Delta)-F\left(\Delta_{1}\right)$, whence by our choice of $k$,

$$
k \eta>-F\left(\Delta_{1}\right) \geq k\left|\Delta_{1}\right|>k(|\Delta|-2 \eta)>k\left(\left|E^{1}\right|-3 \eta\right) .
$$

Thus $\left|E^{1}\right|<4 \eta$, and so $\left|E^{1}\right|=0$. Hence

$$
-\infty<-\overline{(-F)}_{s p} \leq \bar{F}_{s p}<\infty \text {. a.e. on } E \text {. }
$$

For the second part, it is enough now to show that, for any two numbers $c>c_{1}$ we have $|A|=0$ where

$$
A=\left\{\underline{y} ; \underline{y} \in I, \bar{F}_{s p}(\underline{y})>c>c_{1}>-\overline{(-F)}_{s p}(\underline{y})\right\} .
$$

Since here $F$ is MAC-up on $I$, given $\eta>0$ there is an $I$-form $\left\{E_{n}\right\}$ such that $\sum_{n} U\left(F, E_{n} ; I\right)<\eta\left(c-c_{1}\right)$. Then by Lemma 6.5 ,

$$
\begin{aligned}
\left(c-c_{1}\right)|A| & \leq \sum\left(c-c_{1}\right)\left|A \cap E_{n}\right| \leq \sum U\left(F, A \cap E_{n} ; I\right) \\
& \leq \sum U\left(F, E_{n} ; I\right)<\eta\left(c-c_{1}\right) .
\end{aligned}
$$

Thus $|A|<\eta$, and hence $|A|=0$.

Theorem 6.7. Let $F_{s p}^{\prime}=f$ a.e. on $I$. Then there is a set $E \subseteq \stackrel{\circ}{I}$ with $|I \backslash E|=0$ such that, for each $\eta>0$, there is a measurable $E$-form $\mathfrak{E}$ such that

(i) $\left|f(\underline{y})-f\left(\underline{y}^{\prime}\right)\right|<\eta$ for all $\underline{y}, \underline{y}^{\prime}$ in $E_{n}$, and $E_{n}$ has density 1 at each of its points, $n=1, \ldots$, and

(ii) $\left|\frac{1}{|J|} F(J)-f(\underline{y})\right|<\eta$ for every $\mathfrak{E}-$ regular $(\underline{y}, J)$.

Proof. Fix a positive integer $i$. For each $\underline{y}$ in $\stackrel{\circ}{I}$ at which $F_{s p}^{\prime}(\underline{y})=f(\underline{y})$ evidently there is a closed set $A(\underline{y}) \subset \stackrel{\circ}{I}$ having density 1 at $\underline{y}$, such that for 
all $A(y)$-regular $J$ we have

$$
\left|\frac{1}{|J|} F(J)-f(\underline{y})\right|<\frac{1}{i} .
$$

Then, considering the closed sets $A(\underline{y}) \cap J(\underline{y})$ for all such $\underline{y}$ and all cubes $J(\underline{y})$ containing $\underline{y}$, by the Vitali covering theorem there is a sequence $\left\{A\left(\underline{y}_{n}\right) \cap\right.$ $\left.J\left(\underline{y}_{n}\right)\right\}$ such that

$$
\left|I \backslash \bigcup_{n=1}^{\infty} A_{n}\right|=0 \text { where } A_{n}=A\left(\underline{y}_{n}\right) \cap J\left(\underline{y}_{n}\right) .
$$

For each $n$, let $E_{n}^{i}$ denote the set of points $\underline{y}$ of $A_{n}$ at which $A_{n}$ has density 1 and also $F_{s p}^{\prime}(\underline{y})=f(\underline{y})$. Then $\left|A_{n} \backslash E_{n}^{i}\right|=0$, and so $E_{n}^{i}$ is measurable and has density 1 at each of its points and, by the above,

$$
\left|I \backslash E^{i}\right|=0 \text {, where } E^{i}=\bigcup_{n=1}^{\infty} E_{n}^{i} \subseteq \stackrel{\circ}{I} .
$$

Also, since $A\left(\underline{y}_{n}\right)$ is a closed set having density 1 at each point $\underline{y}$ of $E_{n}^{i}$, and for all $A\left(\underline{y}_{n}\right)$-regular $J$ we have

$$
\left|\frac{1}{|J|} F(J)-f\left(\underline{y}_{n}\right)\right|<\frac{1}{i},
$$

the condition $F_{s p}^{\prime}(\underline{y})=f(\underline{y})$ gives, by definition,

$$
\left|f(\underline{y})-f\left(\underline{y}_{n}\right)\right| \leq \frac{1}{i} \text { for all } \underline{y} \text { in } E_{n}^{i}, n=1, \ldots .
$$

Take now $E=\bigcap_{i=1}^{\infty} E^{i}$. Given $\eta>0$, choose $i>2 / \eta$ and set $\mathfrak{E}=\mathfrak{E}^{i} E$. By (11), $E \subseteq \stackrel{\circ}{I}$ and $|I \backslash E|=0$ and $\mathfrak{E}$ is clearly an $E$-form. Then, for each $n$, $\left|E_{n}^{i} \backslash E\right|=0$ and so $E_{n}$ is measurable and has density 1 at each of its points, and by (13) we have $\left|f(\underline{y})-f\left(\underline{y}^{\prime}\right)\right| \leq 2 / i<\eta$ for all $\underline{y}, \underline{y}^{\prime}$ in $E_{n}$. Finally, since $E_{n} \subseteq E_{n}^{i} \subseteq A\left(\underline{y}_{n}\right)$ for all $n$, every E-regular $(\underline{y}, J)$ is both $E_{n}^{i}$-regular, then $\underline{y} \in E_{n}^{i}$, and $A\left(\underline{y}_{n}\right)$-regular for some $n$ and then we have both (12) and (13) and hence $\left|\frac{1}{|J|} F(J)-f(\underline{y})\right|<\frac{2}{i}<\eta$.

Lemma 6.8. If both $F$ and $-F$ are $M A C$-up on $I$, then for every $E_{0} \subset I$, with $\left|E_{0}\right|=0$ and every $\eta>0$ there is an $E_{0}$-form $\mathfrak{E}_{0}$ such that $|F(\Delta)|<\eta$ for every $\mathfrak{E}_{0}$-regular subdivision $\Delta$ in $I$. 
Proof. First we observe the following. Given $\tau>0$, there is an $E_{0}$-form $\mathfrak{E}_{0}$ and a $\delta>0$, such that $F(\Delta)-F\left(\Delta_{1}\right) \leq U\left(F, E_{0} ; I\right)+\tau$ for all pairs of $\mathfrak{E}_{0^{-}}$ regular subdivisions $\Delta, \Delta_{1}$ in $I$ with $|\Delta|-\left|\Delta_{1}\right|<\delta$ and $\Delta_{1} s u b \Delta$. But, since $\left|E_{0}\right|=0$, by Lemma 3.3 (iii) evidently there is an $E_{0}$-form $\mathfrak{E}^{\prime}$ such that $|\Delta|<\delta$ for every $\mathfrak{E}^{\prime}$-regular subdivision $\Delta$. Then $\mathfrak{E}=\mathfrak{E}_{0} \mathfrak{E}^{\prime}$ is an $E_{0}$-form and for every $\mathfrak{E}$-regular subdivision $\Delta$ in $I$ we evidently have $F(\Delta)-F(\emptyset) \leq U\left(F, E_{0} ; I\right)+\tau$.

Now, since $F$ is MAC-up on $I$, by (FDP) there is an $E_{0}$-form $\left\{E_{n}\right\}$ such that $\sum_{n} U\left(F, E_{n} ; I\right)<\eta$. Let $\tau_{n}>0$ be such that $\sum U\left(F, E_{n} ; I\right)+\sum \tau_{n}<$ $\eta$. Since $\left|E_{n}\right|=0$, by above there is an $E_{n}$-form $\mathfrak{E}^{n}$ such that $F\left(\Delta_{n}\right) \leq$ $U\left(F, E_{n} ; I\right)+\tau_{n}$ for all $\mathfrak{E}^{n}$-regular subdivisions $\Delta_{n}$ in $I$.

Let now $\mathfrak{E}$ be an enumeration of the family of sets $\left\{E_{i}^{j} ; i, j=1, \ldots\right\}$. Then $\mathfrak{E}$ is an $E_{0}$-form, and every $\mathfrak{E}$-regular subdivision $\Delta$ in $I$ can be written as $\Delta=\sum_{i=1}^{m} \Delta_{i}$ where $\Delta_{i}$ is $\mathfrak{E}^{i}$-regular, and so

$$
F(\Delta)=\sum_{i=1}^{m} F\left(\Delta_{i}\right) \leq \sum\left(U\left(F, E_{i} ; I\right)+\tau_{i}\right)<\eta .
$$

Similarly, since $-F$ is MAC-up on $I$, there is an $E_{0}$-form $\mathfrak{E}^{\prime}$ such that $-F(\Delta)<$ $\eta$ for every $\mathfrak{E}^{\prime}$-regular subdivision $\Delta$ in $I$. Then $\mathfrak{E}_{0}=\mathfrak{E E}^{\prime}$ is an $E_{0}$-form of the required type.

Theorem 6.9. The following five conditions are equivalent. Also (iii) implies (iv) with any $f=F_{s p}^{\prime}$ a.e. on $I$, and with $\mathfrak{E}$ measurable; and (iv) implies $F_{s p}^{\prime}=f$ a.e. on $I$.

(i) The function $F$ is $M A C$ on $I$.

(ii) Both F and $-F$ are MAC-up on I.

(iii) $F_{s p}^{\prime}$ exists finitely a.e. on $I$, and, for every $E_{0} \subset I$ with $\left|E_{0}\right|=0$ and every $\eta>0$, there is an $E_{0}$-form $\mathfrak{E}_{0}$ such that $|F(\Delta)|<\eta$ for every $\mathfrak{E}_{0}$-regular subdivision $\Delta$ in $I$.

(iv) There is a function $f$ and for each $\eta>0$ there is a $Y$-form $\mathfrak{E}$, such that for every $\mathfrak{E}$-regular division $\left\{\left(\underline{y}_{i}, J_{i}\right)\right\}$ in I we have

$$
\sum_{i}\left|F\left(J_{i}\right)-f\left(\underline{y}_{i}\right)\right| J_{i}||<\eta .
$$


(v) For each $\tau>0$, there is a disjoint, measurable $I$-form $\left\{E_{n}\right\}$ such that, for each $n$ and all $E \subseteq E_{n}$, we have $M(F, E ; I) \leq \tau|E|$.

Proof. From definitions, (i) implies (ii). Also by Theorem 6.6 and Lemma 6.8, (ii) implies (iii). Next, assuming (iii), take any $f=F_{s p}^{\prime}$ a.e. on $I$. By Theorem 6.7, there is a set $E \subseteq \stackrel{\circ}{I}$ with $\left|E_{0}\right|=0$, where $E_{0}=I \backslash E$, and for each $\eta>0$ there is a measurable $E$-form $\mathfrak{E}^{1}$ such that for every $\mathfrak{E}^{1}$-regular $(\underline{y}, J)$ we have

$$
\left|\frac{F(J)}{|J|}-f(\underline{y})\right|<\frac{\eta}{2|I|} .
$$

Also, by (iii) there is an $E_{0}$-form $\mathfrak{E}^{0}$ such that, (separating the positive and negative terms in $F(\Delta)),|F|(\Delta)<\eta / 4$ for every $\mathfrak{E}^{0}$-regular subdivision $\Delta$ in $I$. Further, by Lemma 3.4 there is a $Y$-form $\mathfrak{E}^{\prime}$ such that $|f|\left(\Delta^{*}\right)<\eta / 4$ for every $\mathfrak{E}^{\prime}$-regular division $\Delta^{*}$ over $E_{0}$. Now, take the measurable $Y$-form $\mathfrak{E}$ which is an enumeration of the family of sets $\left\{Y \backslash I, E_{i}^{1}, E_{j}^{0} \cap E_{k}^{\prime} ; i, j, k=1, \ldots\right\}$. Clearly, every $\mathfrak{E}$-regular division $\left\{\left(\underline{y}_{i}, J_{i}\right)\right\}$ in $I$ is of the form $\Delta_{1}^{*}+\Delta^{*}$ where $\Delta_{1}^{*}$ is $\mathfrak{E}^{1}$-regular and $\Delta^{*}$ is both $\mathfrak{E}^{0}$-regular and $\mathfrak{E}^{\prime}$-regular, and hence, then,

$$
\sum\left|F\left(J_{i}\right)-f\left(\underline{y}_{i}\right)\right| J_{i}|| \leq\left|\Delta_{1}\right| \frac{\eta}{2|I|}+|F|(\Delta)+|f|\left(\Delta^{*}\right)<\frac{\eta}{2}+\frac{\eta}{4} 2=\eta .
$$

Thus (iii) implies (iv) in the special way stated.

Next, assuming (iv), to prove (v) we first show that $F_{s p}^{\prime}=f$ a.e. on $I$. For this, it is clearly enough to show that, for each $\eta>0$, we have $|E|<2 \eta$ where

$$
E=\left\{\underline{y} ; \underline{y} \in \stackrel{\circ}{I}, \bar{F}_{s p}(\underline{y})>f(\underline{y})+\eta, o r \overline{(-F)}_{s p}(\underline{y})>\eta-f(\underline{y})\right\} .
$$

Let $\mathfrak{E}^{0}$ be the $Y$-form corresponding to $\eta^{2}$ as furnished by (iv). Let $\mathfrak{E}^{1}=\mathfrak{E}^{0} E$ and let $\xi$ be the family of all $J$ such that, for some $\underline{y},(\underline{y}, J)$ is $\mathfrak{E}^{1}$-regular and $|F(J)-f(\underline{y})| J||>\eta|J|$. By Lemma $6.3, \xi$ is evidently a Vitali $\mathfrak{C}^{1}$-cover. So there is a subdivision $\left\{J_{i}\right\} \subseteq \xi$ in $I$ such that $|E|-\eta<\sum\left|J_{i}\right|$. Then there are points $\underline{y}_{i}$ such that $\left\{\left(\underline{y}_{i}, \bar{J}_{i}\right)\right\}$ is an $\mathfrak{E}^{1}$-regular, and so $\mathfrak{E}^{0}$-regular, division, with $\left|F\left(J_{i}\right)-f\left(\underline{y}_{i}\right)\right| J_{i}||>\eta\left|J_{i}\right|$ for each $i$. Then by our choice of $\mathfrak{E}^{0}$,

$$
\eta(|E|-\eta)<\eta \sum\left|J_{i}\right| \leq \sum\left|F\left(J_{i}\right)-f\left(\underline{y}_{i}\right)\right| J_{i}||<\eta^{2},
$$

whence $|E|<2 \eta$. Now, by Theorem $6.2 f$ is measurable on $I$. So, given $\tau>0$ obviously there is a disjoint, measurable $I$-form $\left\{E_{n}\right\}$ such that, for each $n$, $\left|f(\underline{y})-f\left(\underline{y}^{\prime}\right)\right|<\tau / 2$ for all $\underline{y}, \underline{y}^{\prime}$ in $E_{n}$. Take any $n$ and any $E \subseteq E_{n}$. Given 
$\eta>0$, let $\mathfrak{E}^{0}$ be the $Y$-form as furnished by (iv). Take an open set $G \supseteq E$ with $|G|<|E|+\eta$. By Lemma 3.3 (iii), there is an $E$-form $\mathfrak{E}_{0}$ such that every $\mathfrak{E}_{0}$-regular $J$ is in $G$. Then $\mathfrak{E}^{1}=\mathfrak{E}_{0} \mathfrak{E}^{0}$ is an $E$-form. Take any pair of $\mathfrak{E}^{1}$-regular subdivisions $\Delta=\left\{J_{i}\right\}$ and $\Delta_{1}=\left\{I_{j}\right\}$ in $I$ with $\| \Delta|-| \Delta_{1}||<\eta$. Then $\Delta$ and $\Delta_{1}$ are in $G$, and there are points $\underline{y}_{i}, \underline{x}_{j}$ such that $\left\{\left(\underline{y}_{i}, J_{i}\right)\right\}$ and $\left\{\left(\underline{x}_{j}, I_{j}\right)\right\}$ are $\mathfrak{E}^{1}$-regular and so $\mathfrak{E}^{0}$ regular, divisions in $I$. Fix a point $\underline{y}$ of $E$ and put $m=2+|f(\underline{y})|$. (The case $E=\emptyset$ is trivial.) We have then

$$
\begin{aligned}
\left|F(\Delta)-F\left(\Delta_{1}\right)\right| \leq & \sum\left|F\left(J_{i}\right)-f\left(\underline{y}_{i}\right)\right| J_{i}||+\sum\left|f\left(\underline{y}_{i}\right)-f(\underline{y})\right|\left|J_{i}\right| \\
& +|f(\underline{y})|\left|\sum\right| J_{i}\left|-\sum\right| I_{j}||+\sum\left|f(\underline{y})-f\left(\underline{x}_{j}\right)\right|\left|I_{j}\right| \\
& +\sum\left|f\left(\underline{x}_{j}\right)\right| I_{j}\left|-F\left(I_{j}\right)\right| \\
< & \eta+\sum \frac{\tau\left|J_{i}\right|}{2}+|f(\underline{y})| \eta+\sum \frac{\tau\left|I_{j}\right|}{2}+\eta \\
< & \frac{\tau|G|}{2}+\frac{\tau|G|}{2}+m \eta<\tau|E|+(\tau+m) \eta .
\end{aligned}
$$

Hence, clearly, $M(F, E ; I) \leq \tau|E|$. Thus (iv) implies (v). Since obviously (v) implies (i), the proof is complete.

The notion of $\mathrm{AC}$ is linked with $\mathrm{MAC}$ and $\mathcal{S}$-AC as below.

We call $F$ additive on $I$ if $F(I)=\sum F\left(I_{j}\right)$ for every subdivision $\left\{I_{j}\right\}$ of $I$. This is a hereditary property. For, take any $J$ in $I$, and extend $\{J\}$ to a subdivision $\{J\}+\left\{I_{j}\right\}$ of $I$. Then, for any subdivision $\left\{J_{i}\right\}$ of $J,\left\{J_{i}\right\}+\left\{I_{j}\right\}$ is a subdivision of $I$. So

$$
F(I)=F(J)+\sum F\left(I_{j}\right), \text { and } F(I)=\sum F\left(J_{i}\right)+\sum F\left(I_{j}\right) ;
$$

so $F(J)=\sum F\left(J_{i}\right)$.

Theorem 6.10. If $F$ is additive and $A C$ on $I$, then $F$ is $M A C$ on $I$.

Proof. Since $F$ is AC on $I$, given $\eta>0$ there is a $\delta>0$ such that $\left|F\left(\Delta_{0}\right)\right|<\eta$ for every subdivision $\Delta_{0}$ in $I$ with $\left|\Delta_{0}\right|<\delta$. Let now $\Delta=\left\{J_{i}\right\}$ and $\Delta^{\prime}$ be any two subdivisions in $I$ with $|\Delta|-\left|\Delta^{\prime}\right|<\delta$ and $\Delta^{\prime} s u b \Delta$. We can write $\Delta^{\prime}=\sum_{i} \Delta_{i}$ where $\Delta_{i}$ is in $J_{i}$ for each $i$. Extending $\Delta_{i}$ to a subdivision $\Delta_{i}+\Delta_{i}^{\prime}$ of $J_{i}$, put $\Delta_{0}=\sum_{i} \Delta_{i}^{\prime}$. Then, $\left|\Delta_{0}\right|=|\Delta|-\left|\Delta^{\prime}\right|<\delta$, and so $\left|F\left(\Delta_{0}\right)\right|<\eta$. Since $F$ is additive we get

$$
\left|F(\Delta)-F\left(\Delta^{\prime}\right)\right|=\left|\sum\left(F\left(J_{i}\right)-F\left(\Delta_{i}\right)\right)\right|=\left|\sum F\left(\Delta_{i}^{\prime}\right)\right|=\left|F\left(\Delta_{0}\right)\right|<\eta .
$$

This evidently implies that both $F$ and $-F$ are MAC-up on $I$. Hence by Theorem $6.9, \mathrm{~F}$ is MAC on $I$. 
Theorem 6.11. Let $\mathcal{S}$ consist of all the sifters $s$ for which there is a positive number $c$ such that, for each $y$ in $Y$, we have $s(y, J)=c|J|$ for all $J$ at $y$ in some neighborhood of $y$. If $\bar{F}$ is additive on $I$, then $F$ is $\mathcal{S}$-AC on I if and only if $F$ is $A C$ on $I$.

Proof. Suppose $F$ is $\mathcal{S}$-AC on $I$. Then, given $\eta>0$, there is an $s$ in $\mathcal{S}$ such that $|F(\Delta)|<\eta$ for all $s$-thin divisions $\Delta^{*}$ in $I$. Now, there is a $c>0$ and a gauge $p$ such that, for each $y$ in $Y$ we have $s(y, J)=c|J|$ for all $J$ at $y$ with $d(J)<p(y)$. By Lemma3.3 (i), for any interval $J$, there is a division $\bar{\Delta}^{*}=\left\{\left(\underline{y}_{i}, J_{i}\right)\right\}$ of $J$ such that, for each $i, J_{i}$ is at $\underline{y}_{i}$ and $d\left(J_{i}\right)<p\left(\underline{y}_{i}\right)$, and so $s\left(\underline{y}_{i}, J_{i}\right) \stackrel{\underline{i}_{i}}{=} c\left|J_{i}\right|$. Therefore $s\left(\Delta^{*}\right)=c|\Delta|$.

Consider now any subdivision $\left\{J_{i}\right\}$ in $I$ with $\sum\left|J_{i}\right|<1 / c$. By above, there is a division $\Delta_{i}^{*}$ of $J_{i}$ such that $s\left(\Delta_{i}^{*}\right)=c\left|\Delta_{i}\right|$. Then $\Delta^{*}=\sum_{i} \Delta_{i}^{*}$ is a division in $I$, and it is $s$-thin because $s\left(\Delta^{*}\right)=\sum s\left(\Delta_{i}^{*}\right)=\sum c\left|\Delta_{i}\right|=c \sum\left|J_{i}\right|<1$. So, $\left|\sum F\left(J_{i}\right)\right|=\left|\sum F\left(\Delta_{i}\right)\right|=|F(\Delta)|<\eta$. Hence $F$ is AC on $I$.

The converse is obvious.

\section{$7 \quad$ The $\mathcal{S}$-primitives; Relations between the Integrals}

The following two theorems together characterize the $\mathcal{S}$-integral.

Theorem 7.1. Let $F$ be an additive function of intervals in I. If $F$ is both $\mathcal{S}-A C$ and $M A C$ on $I$, and $F_{s p}^{\prime}=$ fa.e. on $I$, then we have $F=\mathcal{S}(f: I)$.

Proof. Let $\eta>0$. Since $F$ is MAC on $I$ and $f=F_{s p}^{\prime}$ a.e. on $I$, by Theorem 6.9 there is a $Y$-form $\mathfrak{E}$ such that, for every $\mathfrak{E}$-regular division $\left\{\left(\underline{y}_{i}, J_{i}\right)\right\}$ in $I$ we have $\sum\left|F\left(J_{i}\right)-f\left(\underline{y}_{i}\right)\right| J_{i}||<\frac{\eta}{2}$. Again, since $F$ is $\mathcal{S}$-AC on $I$, there is an $s$ in $\mathcal{S}$ such that $\left|F\left(\Delta_{0}\right)\right|<\eta / 2$ for every $s$-thin division $\Delta_{0}^{*}$ in $I$. Now, $\mathfrak{T}=(\mathfrak{E}, s)$ is an $\mathcal{S}$-trimmer. Every $\Delta^{*} \in I(\mathfrak{T})$ is $\mathfrak{E}$-regular and it can be extended to a division $\Delta^{*}+\Delta_{0}^{*}$ of $I$ where $\Delta_{0}^{*}$ is $s$-thin. Then, writing $\Delta^{*}=\left\{\left(\underline{y}_{i}, J_{i}\right)\right\}$ and using additivity of $F$, we have

$$
\left|F(I)-f\left(\Delta^{*}\right)\right| \leq \sum\left|F\left(J_{i}\right)-f\left(\underline{y}_{i}\right)\right| J_{i}||+\left|F\left(\Delta_{0}\right)\right|<\eta .
$$

Hence by Theorem 4.1 (iii), $f$ is $\mathcal{S}$-integrable on $I$ to $F(I)$. Then the proof ends by noting that the hypotheses hold on every $J$ in $I$.

Theorem 7.2. If $F=\mathcal{S}(f: I)$, then $F$ is additive on $I$, and it is both $\mathcal{S}$-AC and $M A C$ on $I$, and further $F_{s p}^{\prime}=f$ a.e. on $I$ and so, by Theorem 6.2, $f$ is measurable on I. 
Proof. By Theorem 4.5, $F$ is additive on $I$. By Theorem 4.1 (ii), given $\eta>0$ there is a $\mathcal{S}$-trimmer $\mathfrak{T}=(\mathfrak{E}, s)$ such that $\mathfrak{T}^{o}(f, I)<\eta$. Then by Lemma 4.6, for every $s$-thin division $\Delta^{*}$ in $I$ we have $|F(\Delta)|<\eta$. Thus $F$ is $\mathcal{S}$-AC on $I$. Again by Lemma 4.6, for every $\mathfrak{E}$-regular division $\left\{\left(\underline{y}_{i}, J_{i}\right)\right\}$ in $I$ we have

$$
\sum\left|F\left(J_{i}\right)-f\left(\underline{y}_{i}\right)\right| J_{i}||<\eta
$$

Hence by Theorem 6.9, $F$ is MAC on $I$ and, further $F_{s p}^{\prime}=f$ a.e. on $I$.

Theorem 7.3. If $f$ is non-negative and $\mathcal{S}$-integrable on $I$, then $f$ is Lebesgue integrable on $I$ to the same value.

Proof. Let $F=\mathcal{S}(f: I)$. Since $f \geq 0$ on $I$, obviously $F(J) \geq 0$ for all $J$ in $I$. Since also $F$ is additive on $I, F$ is of bounded variation on $I$. Therefore $F^{\prime}(y)$ exists finitely a.e. on $I,[19,(5.4)$, p. 115]. Then by Theorem 6.4 and Theorem $7.2 F^{\prime}=F_{s p}^{\prime}=f$ a.e. on $I$, and further, [19, (7.5), p. 119], $F(J) \geq(L) \int_{J} f=G(J)$, say, for all $J$ in $I$. Since $F$ is $\mathcal{S}$-AC on $I$ and $G(J) \leq F(J)$ for all $J$ in $I$, clearly $G$ is $\mathcal{S}$-AC on $I$. Also, $G$ is additive and $\mathrm{AC}$ on $I$, and so by Theorem $6.10 G$ is MAC on $I$, and further $G_{s p}^{\prime}=G^{\prime}=f$ a.e. on $I$. So by Theorem $7.1 G=\mathcal{S}(f: I)$, and hence $G(I)=F(I)$.

Theorem 7.4. For the sifting $\mathcal{S}$ in Theorem 6.11, the $\mathcal{S}$-integral is equivalent to the Lebesgue integral.

Proof. Recalling Remark 4.8, we will consider only finite integrands. First let $F=\mathcal{S}(f: I)$. Then $F$ is additive and $\mathcal{S}$-AC on $I$, and hence by Theorem $6.11 F$ is $\mathrm{AC}$ on $I$, and hence further $F^{\prime}=F_{s p}^{\prime}=f$ a.e. on $I$. Therefore $f$ is Lebesgue integrable on $I$ to $F(I)$.

Conversely, if $f$ has a Lebesgue primitive $F$ on $I$, then $F$ is additive and $\mathrm{AC}$ on $I$. Then by Theorem 6.10 and Theorem $6.11, F$ is MAC and $\mathcal{S}$-AC on $I$; and further $F_{s p}^{\prime}=F^{\prime}=f$ a.e on $I$. Hence $F=\mathcal{S}(f: I)$.

Among the various Henstock- Kurzweil type non-absolute integrals, the HK-integral due to Chew, [7], is very general. It includes the integral of Pfeffer, [17], which in turn includes the generalized Riemann integral, [13]. It is easy to show that, if $F$ is an additive interval function on $I$ such that $C F^{\prime}(\underline{y})=f(\underline{y})$ for all $\underline{y}$ in $I$, then $f$ is HK-integrable on $I$ to $F(I)$. We prove the following assertion.

Theorem 7.5. Let $\mathcal{S}$ consist of all the sifters $s$ for which there is a gauge $q$ such that, for each $\underline{y}$ in $Y$, we have $s(\underline{y}, J)=q(\underline{y})|J|$ for all cubes $J$ at $\underline{y}$ in some neighborhood of $\underline{y}$. Then the $\mathcal{S}$-integral is more general than the $\bar{H} K$ integral. 
Proof. Let $f$ be HK-integrable on $I$ to $c$. From definition, [7, p. 263], given $\eta>0$ there is a gauge $p$ such that $\left|f\left(\Delta^{*}\right)-c\right|<\eta / 2$ for every division $\Delta^{*}$ of $I$ such that $d(J)<p(y)$ and $Q(J)>1-p(y)$ for all $(y, J) \in \Delta^{*}$. Take a gauge $q$ with $q(y)>2|f(y)| / \eta$ for all $y$ in $I$. Define $s(y, J)=q(y)|J|$ if $d(J)<p(y)$ and $Q(J)=1$, and $s(y, J)=1$ otherwise. By Lemma $3.3(\overline{\mathrm{i}})$, there is a $Y$-form $\mathfrak{E}$ such that, for each $n, d\left(E_{n}\right)<p(\underline{y})$ for all $\underline{y}$ in $E_{n}$. Then $\mathfrak{T}=(\mathfrak{E}, s)$ is an $\mathcal{S}$-trimmer.

Now every $\Delta_{1}^{*} \in I(\mathfrak{T})$ is $\mathfrak{E}$-regular and it can be extended to a division $\Delta^{*}=\Delta_{1}^{*}+\Delta_{0}^{*}$ of $I$ with $s\left(\Delta_{0}^{*}\right)<1$. For each $(y, J) \in \Delta_{1}^{*}$, there is an $n$ such that $\underline{y} \in E_{n}$ and $d(J)<d\left(E_{n}\right)$ and $Q(J)>1-\bar{d}\left(E_{n}\right)$, and so $d(J)<p(\underline{y})$ and $Q(J)>1-p \underline{y})$. For each $(\underline{y}, J) \in \Delta_{0}^{*}, \underline{y} \in I$ and $s(\underline{y}, J)<1$, and so

$$
s(\underline{y}, J)=q(\underline{y})|J|>\frac{2}{\eta}|f(\underline{y})||J| ; d(J)<p(\underline{y}) ; Q(J)=1>1-p(\underline{y}) .
$$

Therefore, noting that $\left|f\left(\Delta_{0}^{*}\right)\right| \leq s\left(\Delta_{0}^{*}\right) \eta / 2<\eta / 2$, by above,

$$
\left|f\left(\Delta_{1}^{*}\right)-c\right| \leq\left|f\left(\Delta^{*}\right)-c\right|+\left|f\left(\Delta_{0}^{*}\right)\right|<\frac{\eta}{2}+\frac{\eta}{2}=\eta .
$$

Hence by Theorem 4.1 (iii), $f$ is $\mathcal{S}$-integrable on $I$ to $c$.

\section{The $\mathcal{S}$-integral in $\mathbb{R}$}

In this final section we assume $r=1$, and connect the present theory with other existing theories in one dimension. Given a point function $F$ on $I$, we identify $F$ with the interval function $F([u, v])=F(v)-F(u)$.

Let $E \subseteq I$, and $\delta>0$. Deviating slightly but harmlessly from [22, p. 337], we denote by $V(F, E ; \delta)$ the supremum of $|F(\Delta)|$ for all subdivisions $\Delta$ on $E$ with $|\Delta|<\delta$, and set

$$
V(F, E ; 0)=\inf _{\delta} V(F, E ; \delta), V(F, E)=\sup _{\delta} V(F, E ; \delta) .
$$

We note that $F$ is $\mathrm{VB}$ on $E$ iff $V(F, E)<\infty$, and $\mathrm{AC}$ on $E$ iff $V(F, E ; 0)=0$. If $F$ is $V B$ on each part of some closed $I$-form, then $F$ is called (VBG) on I. If $F$ is continuous on $I$ and $A C$ on each part of some $I$-form, which can then be taken to be closed, then $F$ is called $A C G$ on $I$, [19, p. 223]. The point function $F$ is said to satisfy Lusin's condition $(N)$ on $I$, [19, p. 224], if $|F(H)|=0$ for every $H \subset I$ with $|H|=0$.

The author defined, [22, p. 337], $F$ to be $(P A C)$ on $I$ if for each $\eta>0$ there is an $I$-chain, (see $\S 2),\left\{E_{n}\right\}$ such that $V\left(F, E_{n} ; 0\right)<\eta$ for all $n$; and 
showed that, [22, 3.6, p. 340], $F$ is (PAC) on $I$ iff it satisfies the condition $(\mathrm{N})$ on $I$ and is (VBG) on $I$.

Next, let $O(F ; A)$ denote the oscillation of the point function $F$ on a set $A$. We denote by $V_{*}(F, E ; \delta)$ the supremum of $\sum_{i} O\left(F ; J_{i}\right)$ for all subdivisions $\left\{J_{i}\right\}$ on $E$ with $\sum_{i}\left|J_{i}\right|<\delta$, and set

$$
V_{*}(F, E ; 0)=\inf _{\delta} V_{*}(F, E ; \delta), V_{*}(F, E)=\sup _{\delta} V_{*}(F, E ; \delta) .
$$

If $V_{*}(F, E)<\infty$, then $F$ is called $V B_{*}$ on $E$; if $F$ is $V B_{*}$ on each part of some $I$-form, then $F$ is called $V B G_{*}$ on $I$, [19, p. 228]. If $V_{*}(F, E ; 0)=0$ and $F$ is bounded on $I$, then $F$ is called $A C_{*}$ on $E$; if $F$ is continuous on $I$ and $A C_{*}$ on each part of some $I$-form, which can then taken to be closed, then $F$ is called $A C G_{*}$ on $I ;\left[19,(7.1)\right.$, p. $229 ;(8.8)$, p. 233]. Clearly, $A C G_{*}$ implies ACG, which in turn implies (PAC), [22, 3.5, p. 340].

Theorem 8.1. $F$ is MAC on I iff it is (PAC).

Proof. Suppose $F$ is (PAC) on $I, F$ satisfies the condition $(\mathrm{N})$ on $I$ and is (VBG) on $I$. Let $\left\{A_{n}\right\}$ be a closed $I$-form such that $V\left(F, A_{n}\right)<\infty$ for each $n$. Then $F \mid A_{n}$ has at most countably many points of discontinuity, say $d_{n 1}, d_{n 2}, \ldots$. Given $\eta>0$, for each $d_{n k}$ we can find two intervals $I_{n k}=$ $\left[a_{n k}, d_{n k}\right]$ and $J_{n k}=\left[d_{n k}, b_{n k}\right]$, and two sequences $\left\{I_{n k j}\right\}$ and $\left\{J_{n k j}\right\}$ of nonoverlapping intervals with $\cup_{j} I_{n k j}=\stackrel{\circ}{I}_{n k}$ and $\cup_{j} J_{n k j}=\stackrel{\circ}{J}_{n k}$, such that

$$
\sum_{j} V\left(F, A_{n} \cap I_{n k j}\right)+\sum_{j} V\left(F, A_{n} \cap J_{n k j}\right)<\frac{\eta}{3^{n+k}} .
$$

We note that the set $B_{n}=A_{n} \backslash \bigcup_{k}\left(\stackrel{\circ}{I}_{n k} \cup \stackrel{\circ}{J}_{n k}\right)$ is closed and bounded, and $F \mid B_{n}$ is continuous and VB and satisfies the condition (N) on $B_{n}$. Hence $F$ is $\mathrm{AC}$ on $B_{n},\left[19,(6.7)\right.$, p. 227]. Let then $\delta_{n}>0$ be such that $V\left(F, B_{n} ; \delta_{n}\right)<$ $\eta / 3^{n}$. Now, fix any $n$. Let $\Delta=\left\{J_{i}\right\}$ and $\Delta^{\prime}$ be any two $B_{n}$-regular subdivisions with $|\Delta|-\left|\Delta^{\prime}\right|<\delta_{n}$ and $\Delta^{\prime} s u b \Delta$. We can write $\Delta^{\prime}=\sum_{i} \Delta_{i}$ where $\Delta_{i}$ is in $J_{i}$ for each $i$. Since $B_{n}$ is closed, by $B_{n}$-regularity both $\Delta$ and $\Delta^{\prime}$ are on $B_{n}$. Then, since $B_{n}$ is a linear set, evidently $\Delta_{i}$ can be extended to a subdivision $\Delta_{i}+\Delta_{i}^{\prime}$ of $J_{i}$ where $\Delta_{i}^{\prime}$ is also on $B_{n}$. Let $\Delta_{0}=\sum_{i} \Delta_{i}^{\prime}, \Delta_{0}$ is on $B_{n}$ and $\left|\Delta_{0}\right|=|\Delta|-\left|\Delta^{\prime}\right|<\delta_{n}$. Therefore

$$
\begin{aligned}
\left|F(\Delta)-F\left(\Delta^{\prime}\right)\right| & =\left|\sum\left(F\left(J_{i}\right)-F\left(\Delta_{i}\right)\right)\right| \\
& =\left|\sum F\left(\Delta_{i}^{\prime}\right)\right|=\left|F\left(\Delta_{0}\right)\right| \leq V\left(F, B_{n} ; \delta_{n}\right) .
\end{aligned}
$$


Hence $U\left( \pm F, B_{n} ; I\right) \leq V\left(F, B_{n} ; \delta_{n}\right)$.

Similarly $U\left( \pm F, A_{n} \cap I_{n k j} ; I\right) \leq V\left(F, A_{n} \cap I_{n k j}\right)$ and $U\left( \pm F, A_{n} \cap J_{n k j} ; I\right) \leq$ $V\left(F, A_{n} \cap J_{n k j}\right)$. Hence altogether

$$
\begin{aligned}
\sum U\left( \pm F, B_{n} ; I\right) & +\sum U\left( \pm F, A_{n} \cap I_{n k j} ; I\right)+\sum U\left( \pm F, A_{n} \cap J_{n k j} ; I\right) \\
\leq & \sum V\left(F, B_{n} ; \delta_{n}\right)+\sum V\left(F, A_{n} \cap I_{n k j}\right)+\sum V\left(F, A_{n} \cap J_{n k j}\right) \\
& <\sum \frac{\eta}{3^{n}}+\sum \frac{\eta}{3^{n+k}}<\eta
\end{aligned}
$$

Since the family of sets $\left\{B_{n}, A_{n} \cap I_{n k j}, A_{n} \cap J_{n k j}\right\}$ determines a (closed) $I$ form, it follows that $F$ and $-F$ are MAC-up on $I$. Hence by Theorem $6.9 F$ is MAC on $I$.

Conversely, suppose $F$ is MAC on $I$. By Theorem 6.9, there is a function $f$ and for each $\eta>0$ there is an $\mathbb{R}$-form $\mathfrak{E}^{0}$, such that for every $\mathfrak{E}^{0}$-regular division $\left\{\left(y_{i}, J_{i}\right)\right\}$ in $I$ we have $\sum\left|F\left(J_{i}\right)-f\left(y_{i}\right)\right| J_{i}||<\eta$. Let $E_{n}^{1}=\{y ; y \in$ $I,|f(y)|<n\}$. Then $\mathfrak{E}=\mathfrak{E}^{0} \mathfrak{E}^{1}$ is an $I$-form on each part of which $f$ is bounded. Let $|f|<M_{n}$ on $A_{n}=\cup_{i=1}^{n} E_{i}$. Since $\left\{\bar{E}_{n}\right\}$ is a closed $I$-form, by $\left[22,2.1\right.$, p.337], there is an $I$-chain $\left\{E^{n}\right\}$ such that, for each $n, E^{n}=\cup_{k=1}^{n} E^{k n}$ where $d\left(E^{i n}, E^{j n}\right) \geq 1 / n$ for $i \neq j$ and $E^{k n} \subseteq \bar{E}_{k}$ for $k=1, \ldots, n$. Let $\delta_{n}=\eta /\left(n \eta+M_{n}\right)$.

Consider now any subdivision $\Delta$ on $E^{n}$ with $|\Delta|<\delta_{n}, n$ fixed. If $b_{1}<$ $b_{2}<\cdots<b_{m+1}$ are all the end points of the intervals of $\Delta$, let $J_{p}=\left[b_{p}, b_{p+1}\right]$ for $p=1, \ldots, m$. Let $P=\left\{p ; J_{p} \in \Delta\right\}$. We see that, for each $J \in \Delta$, since $J$ is on $E^{n}$ and $|J|<\delta_{n}<1 / n$, there is a unique $k \leq n$ such that $J$ intersects $E^{k n}$, and then $J$ is on $E^{k n}$. So, for each $p=1, \ldots, m+1$ there is a unique $k_{p} \leq n$ such that $b_{p} \in E^{k_{p} n} \subseteq \bar{E}_{k_{p}}$ and $k_{p}=k_{p+1}$ for all $p$ in $P$. For each $p=1, \ldots, m+1$ we choose $a_{p}=b_{p}$ if $b_{p} \in E_{k_{p}}$, and otherwise we can evidently choose $a_{p} \in E_{k_{p}}$ arbitrarily close to $b_{p}$ such that $I_{p}=\left[a_{p}, b_{p}\right]$ or $\left[b_{p}, a_{p}\right]$ (as the case may be) is $E_{k_{p}}$-regular. Writing $J_{p}^{\prime}=\left[a_{p}, a_{p+1}\right]$ and noting that $k_{p}=k_{p+1}$ for all $p$ in $P$, we thus obtain two $\mathfrak{E}$-regular, and so $\mathfrak{E}^{0}$-regular, divisions $\left\{\left(a_{p}, I_{p}\right) ; a_{p} \neq b_{p}\right\}$ and $\left\{\left(a_{p}, J_{p}^{\prime}\right) ; p \in P\right\}$ in $I$ with $\sum\left|I_{p}\right|<\delta_{n}$ and $\sum_{P}\left|J_{p}^{\prime}\right|<\delta_{n}$, where all $a_{p} \in A_{n}$ since $k_{p} \leq n$. Then, writing $I_{p}=\left[a_{p}, b_{p}\right]$ 
even if $a_{p}=b_{p}$, we have

$$
\begin{aligned}
|F(\Delta)| \leq & \sum_{P}\left|F\left(J_{p}\right)\right| \leq \sum\left(\left|F\left(I_{p+1}\right)\right|+\left|F\left(J_{p}^{\prime}\right)\right|+\left|F\left(I_{p}\right)\right|\right) \\
\leq & \sum\left|F\left(I_{p+1}\right)-f\left(a_{p+1}\right)\right| I_{p+1}||+\sum\left|f\left(a_{p+1}\right)\right|\left|I_{p+1}\right| \\
& +\sum\left|F\left(J_{p}^{\prime}\right)-f\left(a_{p}\right)\right| J_{p}^{\prime}||+\sum\left|f\left(a_{p}\right)\right|\left|J_{p}^{\prime}\right| \\
& +\sum\left|F\left(I_{p}\right)-f\left(a_{p}\right)\right| I_{p}||+\sum\left|f\left(a_{p}\right)\right|\left|I_{p}\right| \\
< & 3 \eta+3 M_{n} \delta_{n}<6 \eta .
\end{aligned}
$$

Therefore, $V\left(F, E^{n} ; 0\right) \leq V\left(F, E^{n} ; \delta_{n}\right) \leq 6 \eta$ for all $n$. Since $\left\{E^{n}\right\}$ is an $I$ chain, $F$ is (PAC) on $I$.

Theorem 8.2. Let $\mathcal{S}$ be the sifting in $\mathbb{R}$ as in Theorem 7.5. Then $F$ is $\mathcal{S}-A C$ on I iff it is $A C G_{*}$ on $I$.

Proof. Suppose $F$ is $\mathrm{ACG}_{*}$ on $I$. Then $F$ is continuous on $I$, and it is $\mathrm{AC}_{*}$ on each part of some closed $I$-form $\left\{E_{n}\right\}$. It is then easy to show that given $\eta>0$, for each $n$ there is $\delta_{n}>0$ such that $|F(\Delta)|<\eta / 2^{n}$ for every subdivision $\Delta$ over $E_{n}$ in $I$ with $|\Delta|<\delta_{n} ;[15,6.4$, p. 27].

Define a sifter $s$ by

$$
s(y, J)= \begin{cases}|J|, & \text { if } y \notin I, \\ |J| / \delta_{n}, & \text { if } y \in E_{n} \backslash \bigcup_{i=1}^{n} E_{i-1}, n=1,2, \ldots ; E_{0}=\emptyset .\end{cases}
$$

Then $s \in \mathcal{S}$. Given any $s$-thin division $\Delta^{*}$ in $I$, put

$$
\Delta_{n}^{*}=\left\{(y, J) ;(y, J) \in \Delta^{*}, y \in E_{n} \backslash \bigcup_{i=1}^{n} E_{i-1}\right\}, n=1,2, \ldots
$$

Then $\left|\Delta_{n}\right|=\delta_{n} s\left(\Delta_{n}^{*}\right) \leq \delta_{n} s\left(\Delta^{*}\right)<\delta_{n}$, and so $|F(\Delta)| \leq \sum\left|F\left(\Delta_{n}\right)\right|<$ $\sum \eta / 2^{n}=\eta$. Hence $F$ is $\mathcal{S}$-AC on $I$.

Conversely, suppose $F$ is $\mathcal{S}$-AC on $I$. Then, given $\eta>0$ there is an $s$ in $\mathcal{S}$ such that $|F(\Delta)|<\eta$ for all $s$-thin divisions $\Delta^{*}$ in $I$. Evidently there is a gauge $p$ on $\mathbb{R}$ such that $s(y, J)<p(y)|J|$ for each $y$ and all $J$ at $y$ with $|J|<1 / p(y)$. Then, for all $(y, J)$ in $I$ with $J$ at $y$ and $|J|<1 / p(y)$, we have $s(y, J)<p(y)|J|<1$, so $\{(y, J)\}$ is $s$-thin, and so $|F(J)|<\eta$. Hence $F$ is continuous on $I$. Now, let $E_{n}=\{y ; y \in I, p(y)<n\},\left\{E_{n}\right\}$ is an $I$-chain. Fix any $n$,and consider any subdivision $\left\{\left[a_{i}, b_{i}\right]\right\}$ on $E_{n}$ with $\sum\left(b_{i}-a_{i}\right)<1 / n$. Let $J_{i}=\left[a_{i}, x_{i}\right] \subseteq\left[a_{i}, b_{i}\right]$. Then $\left|J_{i}\right|<1 / n<1 / p\left(a_{i}\right)$, and so $s\left(a_{i}, J_{i}\right)<p\left(a_{i}\right)\left|J_{i}\right|$, whence $\sum s\left(a_{i}, J_{i}\right) \leq \sum p\left(a_{i}\right)\left|J_{i}\right| \leq \sum n\left(b_{i}-a_{i}\right)<1$. 
Thus $\left\{\left(a_{i}, J_{i}\right)\right\}$ is an $s$-thin division in $I$, and hence $\left|\sum F\left(J_{i}\right)\right|<\eta$. Separating the positive and negative terms $F\left(J_{i}\right)$, we get $\sum\left|F\left(J_{i}\right)\right|<2 \eta$. Hence, clearly $\sum O\left(F ;\left[a_{i}, b_{i}\right]\right) \leq 4 \eta$. Hence $V\left(F, E_{n} ; 0\right) \leq V_{*}\left(F, E_{n} ; 1 / n\right) \leq 4 \eta$ for all $n$. Thus, $F$ is continuous and (PAC) on $I$, and so by $[22,3.5$, p. 340] $F$ is ACG on $I$ and it is also $\mathrm{VBG}_{*}$ on $I$ because each $E_{n}$ is the union of a finite number of sets of diameter less than $1 / n$. Hence, by $\left[19,(8.8)\right.$, p. 233], $F$ is $\mathrm{ACG}_{*}$ on $I$.

Let $I=[a, b]$. We will now identify any $\mathcal{S}$-primitive $F=\mathcal{S}(f: I)$ with the point function $F(x)=\mathcal{S}(f,[a, x]), F(a)=0$.

Theorem 8.3. If $F=\mathcal{S}(f: I)$, then the point function $F$ is Darboux (has the intermediate value property).

Proof. By Theorem 7.2 and Theorem 8.1, $F$ is (PAC) and $\mathcal{S}$-AC on $I$. Being (PAC), $F$ is (VBG) on $I$ and so is Baire-1 on $I$. Also, since $F$ is $\mathcal{S}$-AC, and every $s$ in $\mathcal{S}$ is fine, obviously $F$ lies between its upper and lower one-sided limits on either side everywhere in $I$. Hence by $[2,6.1$, p. 103], $F$ is Darboux on $I$.

Note. Integration by parts formula and mean value theorems can now be proved for the $\mathcal{S}$-integral as in [22, pp. 348-349].

In Theorem 7.4 we showed that, for a suitable $\mathcal{S}$, the $\mathcal{S}$-integral is equivalent to the Lebesgue integral. Now recall that $f$ is restricted Denjoy, $\left(D_{*}\right)$, integrable on $I$ to a number $c$ iff there is a function $F$ on $I$ with $F(a)=0$ and $F(b)=c$ such that $F$ is $\mathrm{ACG}_{*}$ on $I,\left[19\right.$, p. 241]; so then $F^{\prime}(y)$ exists finitely a.e. on $I,\left[19,(7.2)\right.$, p. 230], and $F^{\prime}=f$ a.e. on $I$. Then by Theorem 8.1 and Theorem $6.4, F$ is necessarily MAC on $I$ and $F^{\prime}=F_{s p}^{\prime}$ a.e. on $I$. Hence, recalling Theorem 7.1, Theorem 7.2 and Theorem 8.2, we get at once:

Theorem 8.4. For the sifting $\mathcal{S}$ of Theorem 8.2, the $\mathcal{S}$-integral is equivalent to the $D_{*}$-integral and, hence, to the Perron integral and to the Kurzweil-Henstock integral.

Next recall that $f$ is general Denjoy, $(D)$, integrable on $I$ to a number $c$ iff there is a function $F$ on $I$ with $F(a)=0$ and $F(b)=c$ such that $F$ is ACG on $I,\left[19\right.$, p. 241]; so then $F_{a p}^{\prime}(y)$ exists finitely a.e. on $I$, [19, $(4.3)$, p. 222], and $F_{a p}^{\prime}=f$ a.e. on $I$.

Theorem 8.5. Let $\mathcal{S}$ consist of all the sifters $s$ in $\mathbb{R}$ which are continuous in the sense that, for each $y$ in $\mathbb{R}, s(y,[y, x]) \rightarrow 0$ as $x \rightarrow y+$, and $s(y,[x, y]) \rightarrow 0$ as $x \rightarrow y-$. Then the $\mathcal{S}$-integral is equivalent to the $D$-integral. 
Proof. Suppose $f$ is D-integrable on $I$ with primitive $F$. Since $F$ is ACG on $I$, it is (PAC) on $I$ and so by Theorem 8.1 it is MAC on $I$. Then, recalling Theorem 6.6, we have $F_{s p}^{\prime}=F_{a p}^{\prime}=f$ a.e. on $I$. Also, since $F$ is continuous, given $\eta>0$ clearly there is an $s$ in $\mathcal{S}$ such that $s(y, J)=|F(J)| / \eta$ for all $(y, J)$ in $I$, which trivially implies that $F$ is $\mathcal{S}$-AC on $I$. Hence by Theorem $7.1 f$ is $\mathcal{S}$-integrable on $I$ to $F(I)=F(b)$.

Conversely, let $\mathrm{F}=\mathcal{S}(f: I)$. By Theorem 7.2 and Theorem 8.1, $F$ is $\mathcal{S}$-AC and $(\mathrm{PAC})$ on $I$, and $F_{s p}^{\prime}=f$ a.e. on $I$. Since every $s$ in $\mathcal{S}$ is continuous, the condition $\mathcal{S}$-AC clearly implies that $F$ is continuous on $I$. Thus $F$ is continuous and (PAC) on $I$ and hence by $[22,3.5$ p. 340] $F$ is ACG on $I$ and obviously $F_{a p}^{\prime}=F_{s p}^{\prime}=f$ a.e. on $I$. Hence $f$ is D-integrable on $I$ to $F(b)=F(I)$.

We will conclude this paper by indicating the extent of the integrals in the $\mathcal{S}$-scale. In terms of an abstract limit process $\mathcal{T}$ in $\mathbb{R}$, the author defined the $(\mathcal{T P})$ and $(\mathcal{T} \mathrm{D})$ integrals, which are equivalent, [20, 21]. By [20, 4.4, p. 242] and $[21,5.1$, p. 303], the function $f$ is $(\mathcal{T} P)$ or $(\mathcal{T} D)$-integrable on $I$ to a number $c$ iff there is a function $F$ on $I$ with $F(I)=c$, such that $F$ is $\mathcal{T}$-continuous and (PAC) on $I$ and $F_{a p}^{\prime}=f$ a.e. on $I$. If $\mathcal{T}$ is the approximate limit process then this integral is substantially more general than the D-integral and the various approximately continuous integrals, [22, p. 352]. Now, let $\mathcal{B}$ be Thomson's simple (local) system that is bilateral and filtering down, $\mathcal{B}$ induces a unique limit process, $\mathcal{T}$ say, in the natural way, [25, pp. 280-282]. Let $\mathcal{S}$ consist of all the sifters $s$ in $\mathbb{R}$ which are $\mathcal{T}$-continuous, (i.e $\mathcal{B}$-continuous), in the sense that, for each $y$ in $\mathbb{R}$

$$
\mathcal{T}-\lim _{x \rightarrow y-} s(y,[x, y])=\mathcal{T}-\lim _{x \rightarrow y+} s(y,[y, x])=0 .
$$

Then $\mathcal{S}$ is a sifting and, as in Theorem 8.5, the $\mathcal{S}$-integral is equivalent to the $(\mathcal{T P})$ and $(\mathcal{T} \mathrm{D})$ integrals. This integral is more general than the $\mathcal{B}$-integral defined by Wang \& Ding, [26], in special cases.

Next, given any sifting $\mathcal{S}$ in $\mathbb{R}, \mathcal{S}$ induces a unique limit process, $\mathcal{T}$ say, defined by weakening $\mathcal{S}$-AC in the natural way. Then the $\mathcal{S}$-integral is included in the $(\mathcal{T P})$ and $(\mathcal{T D})$ integrals.

Finally, given any limit process $\mathcal{T}$, let $f$ be $(\mathcal{T P})$ or $(\mathcal{T} \mathrm{D})$-integrable on $I$ with primitive $F, F(x)=0$ for $x \leq a$ and $F(x)=F(b)$ for $x \geq b$. Then $F$ is $\mathcal{T}$-continuous and (PAC), and so (MAC) on $I$, and $F_{s p}^{\prime}=F_{a p}^{\prime}=f$ a.e. on $I$. Also, by $[20,4.4 .1$, p. 242] $F$ is Darboux on $I$, which plainly implies that, if $\mathcal{S}$ consists of all the sifters $s$ in $\mathbb{R}$ for which there is a $c>0$ such that, for each $y$ in $\mathbb{R}, s(y, J)=c|F(J)|$ for all $J$ at $y$ in some neighborhood of $y$, then $\mathcal{S}$ is a sifting (depending on $f$ ), and $F$ is trivially $\mathcal{S}$-AC on $I$. Hence $f$ is $\mathcal{S}$-integrable on $I$ to $F(b)$. Thus the integrals in the Riemann $\mathcal{S}$-scale and in 
the Perron-Denjoy $\mathcal{T}$-scale differ only in classification, and not in extent.

\section{References}

[1] Li Baoling, Convergence theorems for the variational integral, Real Analysis Exch., 15 (1989-1990), 324-332.

[2] A. M. Bruckner and J. G. Ceder, Darboux continuity, Jber. Deutsch. Math- Verein, 67 (1965), 93-117.

[3] P. S. Bullen, A constructive definition of an integral, Tôhoku Math. J., 22 (1970), 597-603.

[4] P. S. Bullen and C. M. Lee, On the integrals of Perron type, Trans. Amer. Math. Soc., 182 (1973), $481-501$.

[5] P. S. Bullen, D. N. Sarkhel and R.Výborný, A Riemann view of the Lebesgue integral, South East Asian Bull. Math. , 12 (1988), 39-51.

[6] V. G. Chelidze and A. G. Dzhvarsheishvili, The Theory of the Denjoy Integral and Some Applications, translated by P. S. Bullen, World Scientific, Singapore, 1989.

[7] Chew Tuan Seng, On the equivalence of Henstock-Kurzweil and restricted Denjoy integrals in $\mathbb{R}^{n}$, Real Analysis Exch., 15 (1989-1990), 259-268.

[8] R. B. Darst and E .J. McShane, The deterministic Itô-belated integral is equivalent to the Lebesgue integral, Proc. Amer. Math. Soc., 72 (1978), $271-275$.

[9] Russell A. Gordon, Equivalence of the generalized Riemann and restricted Denjoy integrals, Real Analysis Exch., 12 (1986-1987), 551-574.

[10] Russell A. Gordon, Another look at a convergence theorem for the Henstock integral, Real Analysis Exch., 15 (1989-1990), 724-728.

[11] Russell A. Gordon, Another approach to the controlled convergence theorem, Real Analysis Exch., 16 (1990-1991), 306-310.

[12] R. Henstock, The variation on the real line, Proc. Roy. Irish Acad. Sect.A, 79 (1979), \#11, 1-10.

[13] R. Henstock, Lectures on the Theory of Integration, World Scientific, Singapore, 1988. 
[14] J. Kurzweil, Nichtabsolut Konvergente Integrale,Leipzig, 1980.

[15] Lee Peng Yee, Lanzhou Lectures on Henstock Integration, World Scientific, Singapore, 1989.

[16] E. J. McShane, Unified Integration,New York, 1983.

[17] W. F. Pfeffer, The divergence theorem, Trans. Amer. Math. Soc., 295 (1986), 665-685.

[18] W. F. Pfeffer and W. Yang, A multidimensional variational integral and its extensions, Real Analysis Exch., 15 (1989-1990),111-169.

[19] S. Saks, Theory of the Integral, Dover, New York, 1964.

[20] D. N. Sarkhel, A wide Perron integral, Bull. Austral. Math. Soc., 34 (1986), 233-251.

[21] D. N. Sarkhel, A wide constructive integral, Math. Japon., 32 (1987), 295-309.

[22] D. N. Sarkhel and A. B. Kar, (PVB) functions and integration, J. Austral. Math. Soc., Ser.A, 36 (1984), 335-353.

[23] Lu Shi-Pan and Liao Kee Cheng, On generalized dominated convergence, Real Analysis Exch., 16 (1990-1991), $74-78$.

[24] B. S. Thomson, Constructive definitions for non-absolutely convergent integrals, Proc. London Math. Soc., (3) 20 (1970), 699-716.

[25] B. S. Thomson, Derivation bases on the real line. (I), (II), Real Analysis Exch., 8 (1982-1983), 67-207, 278-442.

[26] Wang Cai Shi and Ding Chuan Song, An integral involving Thomson's local systems, Real Analysis Exch., 19 (1993-1994), 248-253. 